\title{
EXTENDING ŁUKASIEWICZ LOGICS WITH A MODALITY: ALGEBRAIC APPROACH TO RELATIONAL SEMANTICS
}

\author{
GEORGES HANSOUL AND BRUNO TEHEUX
}

\begin{abstract}
This paper presents an algebraic approach of some many-valued generalizations of modal logic. The starting point is the definition of the [0,1]-valued KRIPKE models, where $[0,1]$ denotes the well known MV-algebra.

Two types of structures are used to define validity of formulas: the class of frames and the class of $\mathrm{E}_{n}$-valued frames. The latter structures are frames in which we specify in each world $u$ the set (a subalgebra of $t_{n}$ ) of the allowed truth values of the formulas in $u$.

We apply and develop algebraic tools (namely, canonical and strong canonical extensions) to generate complete modal $n+1$-valued logics and we obtain many-valued counterparts of SHALQVIST canonicity result.
\end{abstract}

\section{INTRODUCTION}

When one looks backwards in the history of modern logic, one can notice that modal logics and many-valued logics are born approximatively at the same time. It even appears that some logicians, such as ŁUKASIEWICZ, defined many-valued systems in order to deal with modalities (see chapter 21 of [20]). By considering a third truth value, they meant to express that a formula can, for example, be possible without being true.

Nevertheless, these two types of formalisms followed their own ways. They are indeed two generalizations of propositional calculus with very different properties.

On the one hand, mathematicians studied many-valued logics (as defined by J. LuKAsIEwICZ in [27; see 28] for an English translation and 8] for a monograph on the subject) through their algebraic form: the class of MV-algebras that was define d by C.C. ChAng in 1958 (see [5] and [6]) in order to obtain an algebraic proof of the completeness result for the infinite-valued ŁUKASIEWICZ logic.

On the other hand, modal logics were also studied through their algebraic disguises, which are the Boolean algebras with operators (introduced in [24] and [25]). But the success of modal logics among the communities of mathematicians, computer scientists and philosophers is a consequence of the relational semantics introduced in the sixties by S. KRIPKE (see [26]). With KRIPKE semantic, also called possible worlds semantic, a formula is possible in a world $w$ if there is a world accessible to $w$ in which the formula is true. From then on, in their approach of modal logic, mathematicians have been focusing their attention on the connection between the algebraic and the relational semantics. This approach allowed a great simplification of the proof of the completeness of normal modal logics through the construction of the so-called canonical model (see [32] for example).

2000 Mathematics Subject Classification. 03B45, 03B50.

Key words and phrases. modal logic, many-valued logic, KRIPKE semantic, relational semantic, canonical model, MV-algebras. 
This paper presents some many-valued generalizations of modal logic. Many authors have already initiated such studies (see [10, 11, 12, 30, 2, 3]). As each of these authors realized, since the success of modal logic is a consequence of its KRIPKE semantic, it is wise to consider this semantic as a starting point for many-valued generalizations of modal logic.

The diversity of the many-valued modal systems that have already been introduced proves that the principle of "keeping KRIPKE semantic" still allows a lot of freedom in the definitions. Indeed, there are many ways for the generalization of KRIPKE models to a many-valued realm. Nevertheless, these generalizations can be classified in two (non exclusive) classes: the class of the KRIPKE models in which propositional variables are evaluated in a set with more than two elements and the class of the KRIPKE models in which the accessibility relation is many-valued.

Facing these possibilities, the logician may combine several criteria to determine the approach he wants to follow. His choice can be guided by the applications he wishes to develop for his systems (as in [12]), by the theme of the results that are to be obtained in priority (translation between modal formulas and first order formulas for example), by the tools he wishes to apply (algebras, coalgebras, model theory, ...), by his intuition and his abilities.

In our case, we were guided by the will to consider many-valued KRIPKE models for which the existing algebraic tools could be applied or generalized. Hence, we have decided to base our approach on ŁUKASIEWICZ logics. The KRIPKE models that we consider are models with a crisp relation in which variables have their truth value in the MV-algebra $[0,1]$. Since the variety of MV-algebras shares a lot of properties with the variety of Boolean algebras, we hoped to find in this variety the properties required for an algebraic approach that would lead, at least, to a completeness result.

The first part of the paper, sections $2-6$, contains essentially the results of an unpublished paper [21] of the authors. In this part, we define the [0,1]-valued KRIPKE models and their corresponding modal many-valued logics. We then define the varieties of the modal manyvalued algebras that give an algebraic semantic with respect to which any modal many-valued logic is complete. This result is a step towards the construction of the canonical model of any modal many-valued logic and towards Proposition 5.6 that shows that the valuation map in the canonical model extends nicely to formulas. This construction leads us to completeness results for the modal many-valued logics and KRIPKE models, i.e., to syntactic characterizations of the smallest modal many-valued logic (Proposition 6.4 and Proposition 6.6). In this regard, the reader may feel a bit unsatisfied with the results we obtain about infinitely valued logics since these results involve an infinitary deduction rule.

The second part of the paper focuses on the problem of completeness of modal $n+1$-valued logics with regard to classes of structures. Two types of structures are considered, giving rise to two types of completeness: the frames and strong KRIPKE completeness on the one hand and the $\mathrm{E}_{n}$-valued frames and KRIPKE completeness on the other hand. A frame is a structure with a single binary relation (the accessibility relation). An $\mathrm{E}_{n}$-valued frame (where $\mathrm{E}_{n}$ is the subalgebra $\left\{0, \frac{1}{n}, \ldots, \frac{n-1}{n}, 1\right\}$ of $[0,1]$ for any strictly positive integer $\left.n\right)$ is a frame in which we have specified in each world the set of the allowed truth values (a subalgebra of $t_{n}$ ) for the propositions in this world.

These completeness problems are approached in a syntactic way with the algebraic tool. In section 8 , we obtain KRIPKE completeness results by studying preservation of equations through canonical extensions (algebras are considered as expanded distributive lattices). We obtain a many-valued equivalent (Theorem 8.26) of the SAHLQVIST canonicity result by slightly adapting the proof of the corresponding result for Boolean modal logic that is presented in [32]. 
Strong KRIPKE completeness results are then obtained in section 9 by studying preservation of equations through a new type of extension, called the strong canonical extension. The main result of this section is Theorem 9.16 that identifies a subfamilly of SAHLQVIST equations that are preserved under strong canonical extension and thus, that define strongly canonical logics.

\section{A Relational Semantic For modal $[0,1]$-Valued logics}

Most of the authors who have recently studied modal extensions of many-valued systems agree on the necessity to develop systems that admit a Kripke style semantic (see [2, 10, 11, 30]). We follow that reasonable rule and start by introducing a suitable notion of KRIPKE model.

Let us denote by Prop an infinite set of propositional variables, by $\mathcal{L}_{M V}$ the language $\{\rightarrow, \neg\}$ where $\rightarrow$ is binary and $\neg$ is unary, by $\square$ a unary symbol and by Form the set of formulas defined inductively by the following rules:

(1) Prop $\subseteq$ Form;

(2) if $\phi$ and $\psi$ are in Form then $\neg \phi, \phi \rightarrow \psi$ and $\square \phi$ are in Form.

The intended meaning of $\phi \rightarrow \psi$ and $\neg \psi$ is clear (these formulas have their usual EuKASIEwICZ meaning) and $\square \phi$ can be read, for example, as "necessarily $\phi$ ".

In the sequel, we use some standard abbreviations: the formula $\phi \oplus \psi$ stands for $\neg \phi \rightarrow \psi$, the formula $\psi \odot \phi$ for $\neg(\neg \psi \oplus \neg \phi)$, the formula $\phi \vee \psi$ for $(\phi \rightarrow \psi) \rightarrow \psi$, the formula $\phi \wedge \psi$ for $\neg(\neg \phi \vee \neg \psi)$ and $\diamond \phi$ for $\neg \square \neg \phi$.

The classical definition of a KRIPKE model can easily be extended to a $[0,1]$-valued realm. We consider that the real unit interval $[0,1]$ is endowed with the LUKASIEWICZ implication and negation: $x \rightarrow y=\min (1,1-x+y)$ and $\neg x=1-x$. If $n$ is a strictly positive integer, we denote by $\mathrm{E}_{n}$ the subset $\left\{0, \frac{1}{n}, \ldots, \frac{n-1}{n}, 1\right\}$ of $[0,1]$. Note that $\mathrm{E}_{n}$ is closed for $\rightarrow$ and $\neg$.

Definition 2.1. A many-valued KRIPKE model (or simply a many-valued model) $\mathcal{M}=\langle W, R$, Val $\rangle$ is given by a non empty set $W$, an accessibility relation $R \subseteq W \times W$ and a map Val : $W \times$ Prop $\rightarrow$ $[0,1]$. If $n$ is a positive integer such that $\operatorname{Val}(W, \operatorname{Prop}) \subseteq \mathrm{E}_{n}$, then we call $\mathcal{M}$ an $n+1$-valued KRIPKE model.

A frame $\mathfrak{F}=\langle W, R\rangle$ is given by an non empty set $W$ and an accessibility relation $R$ on $W$. A model $\mathcal{M}=\left\langle W^{\prime}, R^{\prime}\right.$, Val $\rangle$ is based on the frame $\mathfrak{F}=\langle W, R\rangle$ if $W=W^{\prime}$ and $R=R^{\prime}$.

If $\mathcal{M}=\langle W, R$, Val $\rangle$ is a many-valued KRIPKE model, we extend inductively the map Val to formulas of Form by the following rules:

- $\operatorname{Val}(w, \phi \rightarrow \psi)=\operatorname{Val}(w, \phi) \rightarrow \operatorname{Val}(w, \psi)$ and $\operatorname{Val}(w, \neg \phi)=\neg \operatorname{Val}(w, \phi)$,

- $\operatorname{Val}(w, \square \phi)=\bigwedge\left\{\operatorname{Val}\left(w^{\prime}, \phi\right) \mid\left(w, w^{\prime}\right) \in R\right\}$,

for any formulas $\phi$ and $\psi$ of Form and any world $w$ of $W$ (where $\Lambda$ is the infimum in $[0,1]$ ).

We write $\mathcal{M}, w \models \phi$ or simply $w \models \phi$ (and say that $\phi$ is true at $w$ ) whenever $\operatorname{Val}(w, \phi)=1$, and $\mathcal{M} \models \phi$ whenever $w \models \phi$ for any $w$ in $W$. In that case, we say that $\phi$ is true in $\mathcal{M}$. If $\Gamma$ is a set of formulas that are true in a model $\mathcal{M}$, then $\mathcal{M}$ is a model of $\Gamma$. If $\mathfrak{F}$ is a frame and $\phi$ is a formula that is true in any model based on $\mathfrak{F}$, we say that $\phi$ is valid in $\mathfrak{F}$ and write $\mathfrak{F}=\phi$. If $\phi$ is true in any $n+1$-valued model based on $\mathfrak{F}$, we write $\mathfrak{F} \models_{n} \phi$ (or even $\mathfrak{F} \models \phi$ if the context is clear).

Note that the 2-valued KRIPKE models coincide with the KRIPKE models of normal modal logics (then the operation $\oplus$ matches up with the supremum $\vee$ ). More general KRIPKE models, in which the accessibility relation can be many-valued, are considered in [2. In the sequel, we prove that there is another class of structures that turns out to be richer than the class of frames. 
Proposition 2.2. If $\tau$ is an increasing unary term of the language $\mathcal{L}_{M V}$ (i.e., a unary term whose interprepation on any $M V$-algera is an increasing map), then the formulas

$$
\square(p \rightarrow q) \rightarrow(\square p \rightarrow \square q), \quad \square(p \wedge q) \leftrightarrow \square p \wedge \square q, \quad \square \tau(p) \leftrightarrow \tau(\square p)
$$

are tautologies, i.e., they are true in any many-valued KRIPKE model.

The idea of using these models as a semantic for modal many-valued logics is not new. See [30] for example.

\section{3. ŁUKASIEWICZ MODAL MANY-VALUED LOGICS}

The purpose of this section is to introduce a family of modal many-valued logics and their corresponding algebras in order to tackle completeness results through canonicity. We refer to [8, 22] for an introduction to ŁukAsiewicz logic and to [1, 4, 7, for an introduction to modal logic.

The modal theory of a frame is captured in the concept of a modal many-valued logic.

Definition 3.1. A modal many-valued logic is a set $\mathbf{L}$ of formulas of Form that is closed under modus ponens, uniform substitution, the necessitation rule (RN) (if $\phi \in \mathbf{L}$ then $\square \phi \in \mathbf{L}$ ) and that contains

- an axiomatic base of ŁUKASIEwICZ many-valued logic $(p \rightarrow(q \rightarrow p),(p \rightarrow q) \rightarrow((q \rightarrow$ $r) \rightarrow(p \rightarrow r)),((p \rightarrow q) \rightarrow q) \rightarrow((q \rightarrow p) \rightarrow p),(\neg p \rightarrow \neg q) \rightarrow(q \rightarrow p)$ for example);

- the axiom (K) of modal logic: $\square(p \rightarrow q) \rightarrow(\square p \rightarrow \square q)$,

- the formulas $\square(p \oplus p) \leftrightarrow \square p \oplus \square p$ and $\square(p \odot p) \leftrightarrow \square p \odot \square p$,

- the formula $\square\left(p \oplus p^{m}\right) \leftrightarrow\left(\square p \oplus(\square p)^{m}\right)$ for every positive integer $m$.

As usual, we write $\vdash_{\mathbf{L}} \phi$ and say that $\phi$ is a theorem of $\mathbf{L}$ whenever $\phi \in \mathbf{L}$ and denote by $\mathbf{K}$ the smallest modal many-valued logic. If in addition $\mathbf{L}$ contains an axiomatic base of the $n+1$-valued ŁUKASIEWICZ logic, we say that $\mathbf{L}$ is a modal $E_{n}$-valued logic and we denote by $\mathbf{K}_{n}$ the smallest of these logics.

It is easy to prove that if $\mathcal{C}$ is a class of frames, then the modal theory of $\mathcal{C}$ (i.e., the set of formulas that are true in any model based on a frame of $\mathcal{C}$ ) is a modal many-valued logic.

Note that, according to Proposition 2.2 many-valued KRIPKE models form a sound semantic for $\mathbf{K}$. Let us also remark that, as it will appear clearly in the sequel of the paper (in the proof of Proposition 5.6), we only use the last family of axioms as a kind of conservative law for $\square$ with respect to infinitely great elements. Moreover Proposition 6.4 provides an axiomatization of the finitely-valued logics without this family of axioms (and this explains why we have added the axiom $\square(p \oplus p) \leftrightarrow \square p \oplus \square p$ even if it is equivalent to $\square\left(p \oplus p^{m}\right) \leftrightarrow\left(\square p \oplus(\square p)^{m}\right)$ with $\left.m=1\right)$.

We can easily obtains the following theorems and admissible rules of $\mathbf{K}$.

Proposition 3.2. The following formulas are theorems of $\mathbf{K}$ :

$$
\begin{array}{ll}
\square(p \rightarrow q) \rightarrow(\diamond p \rightarrow \diamond q), & \nabla(p \oplus q) \rightarrow(\diamond p \oplus \diamond q), \\
(\square p \wedge \diamond q) \rightarrow \diamond(p \wedge q), & \square(p \wedge q) \rightarrow(\square p \wedge \square q) \\
(\square p \odot \square q) \rightarrow \square(p \odot q) . &
\end{array}
$$

Moreover, the following rules are derivable in $\mathbf{K}$ :
(1) $\frac{\phi \leftrightarrow \psi}{\square \phi \leftrightarrow \square \psi}$,
(2) $\frac{\phi_{1} \odot \cdots \odot \phi_{n} \rightarrow \psi}{\square \phi_{1} \odot \cdots \odot \square \phi_{n} \rightarrow \square \psi}$. 
Proof. The proofs are simple adaptations of the two-valued proofs.

Note that at this point of our development, we are not able to determine if the formula $(\square p \wedge \square q) \rightarrow \square(p \wedge q)$ is in $\mathbf{K}$ or in $\mathbf{K}_{n}$ (for $\left.n \geq 2\right)$. We shall conclude latter, thanks to a completeness result, that it is a theorem of $\mathbf{K}_{n}$ for any $n$. On the contrary, the formula $\square(p \odot q) \rightarrow \square p \odot \square q$ is not a theorem of $\mathbf{K}_{n}$ for any $n \geq 2$ since it is not a $\mathbf{K}_{n}$-tautology.

Definition 3.3. If $\Gamma \cup\{\phi\}$ is a set of formulas and if $\mathbf{L}$ is a many-valued modal logic, we say that $\phi$ is deducible from $\Gamma$ in $\mathbf{L}$ and write $\Gamma \vdash_{\mathbf{L}} \phi$ (or simply $\Gamma \vdash \phi$ when $\mathbf{L}=\mathbf{K}$ or $\mathbf{L}=\mathbf{K}_{n}$ according to the context) if there is a finite subset $\left\{\phi_{1}, \ldots, \phi_{r}\right\}$ of $\Gamma$ and some positive integers $m_{1}, \ldots, m_{r}$ such that $\vdash_{\mathbf{L}}\left(\phi_{1}^{m_{1}} \odot \cdots \odot \phi_{r}^{m_{r}}\right) \rightarrow \phi$.

\section{Modal many-Valued algebras And the Algebraic semantic}

We introduce the varieties of modal many-valued algebras and state the completeness result for modal many-valued logics and algebras. This somehow obvious result can be seen as a step towards the construction of the canonical model and possible completeness theorems for manyvalued modal logics and many-valued KRIPKE models. We refer the reader to [8] or [18, for an introduction to the variety of MV-algebras.

Definition 4.1. If $\mathbf{L}$ is a modal many-valued logic then an $\mathbf{L}$-algebra is an algebra $A$ over the language $\mathcal{L}_{M M V}=\{\rightarrow, \neg, \square, 0,1\}$ that satisfies the equations naturally induced by the formulas of $\mathbf{L}$. We denote by $\mathcal{M M V}$ (resp. $\mathcal{M M \mathcal { V } _ { n }}$ ) the variety of $\mathbf{K}$-algebras (resp. the variety of $\mathbf{K}_{n^{-}}$ algebras). Members of $\mathcal{M M V}$ (resp. $\mathcal{M M V}_{n}$ ) are simply called modal many-valued algebras or MMV-algebras (resp. modal $E_{n}$-valued algebras or $\mathrm{MMV}_{n}$-algebras) and the operation $\square$ is called a dual operator.

A modal many-valued logic $\mathbf{L}$ is often given by a set $\Gamma$ of axioms, i.e., the logic $\mathbf{L}$ is defined as the smallest modal many-valued logic that contains $\mathbf{K} \cup \Gamma$, and is denoted by $\mathbf{K}+\Gamma$.

Since the most commonly used axiomatization of the variety $\mathcal{M V}$ of MV-algebras is given over the language $\{\oplus, \odot, \neg, 0,1\}$, we preferably use this language instead of $\mathcal{L}_{M V}$ (with the help of the equation $(\neg \phi \oplus \psi)=(\phi \rightarrow \psi)$ that defines $\rightarrow$ from $\{\neg, \oplus\}$ in $\mathcal{M V})$. Thus, an MMV-algebra is an algebra $A=\langle A, \oplus, \odot, \neg, \square, 0,1\rangle$ of type $(2,2,1,1,0,0)$ such that

- the reduct of $A$ to the language $\{\oplus, \odot, \neg, 0,1\}$ is an MV-algebra (i.e., A satisfies the equations $\neg \neg x=x, x \oplus 1=1, \neg 0=1, x \odot y=\neg(\neg x \oplus \neg y),(x \odot \neg y) \oplus y=(y \odot \neg x) \oplus x)$;

- the algebra $A$ satisfies the equations $\square(x \rightarrow y) \rightarrow(\square x \rightarrow \square y)=1, \square(x \oplus x)=\square x \oplus \square x$, $\square(x \odot x)=\square x \odot \square x$ and $\square\left(x \oplus x^{m}\right)=\left(\square x \oplus(\square x)^{m}\right)$ for every positive integer $m$.

Similarly, an $\mathrm{MMV}_{n}$-algebra is an MMV-algebra whose reduct to the language of MV-algebras is a member of the variety $\mathbb{H} \mathbb{S P}\left(\mathrm{七}_{n}\right)=\mathbb{I S P}\left(\mathfrak{七}_{n}\right)$.

Recall that on an MV-algebra $A$, the relation $\leq$ defined by

$$
x \leq y \text { if } x \rightarrow y=1
$$

is a bounded distributive lattice order on $A$ with $x \vee y=(x \rightarrow y) \rightarrow y$ and $x \wedge y=\neg(\neg x \vee \neg y)$.

It is not the object of this paper to study the varieties of MMV-algebras in details.

Definition 4.2. A filter of an MMV-algebra $A$ is a filter of its MV-algebra reduct (i.e., a non empty increasing subset of $A$ that contains $y$ whenever it contains $x$ and $x \rightarrow y$ ). If $X$ is a subset of $A$, we denote by $\langle X\rangle$ the filter generated by $X$, i.e., the smallest filter of $A$ that contains $X$. 
We denote by $\mathcal{F}_{\mathbf{L}}$ the the Lindenbaum - Tarski algebra of $\mathbf{L}$, i.e., the set of formulas modulo L-equivalence. If $\phi$ is a formula, we denote by $\phi^{\mathbf{L}}$ its class in $\mathcal{F}_{\mathbf{L}}$. If $\Gamma$ is a set of formulas, we denote by $\Gamma^{\mathbf{L}}$ the set $\left\{\phi^{\mathbf{L}} \mid \phi \in \Gamma\right\}$.

Recall that the lattice of filters of an MV-algebra $A$ is isomorphic to the lattice of congruences of $A$. The congruence $\theta_{F}$ associated to a filter $F$ by this isomorphism is defined by $(x, y) \in \theta_{F}$ if $(x \rightarrow y) \odot(y \rightarrow x) \in F$. As usual, we denote by $A / F$ the quotient $A / \theta_{F}$.

For our purpose, the next result is fundamental, albeit an obvious one.

Lemma 4.3. If $\mathbf{L}$ is a modal many-valued logic, and $\Gamma \cup\{\phi\}$ is a set of formulas then $\Gamma \vdash_{\mathbf{L}} \phi$ if and only if $\left(\phi^{\mathbf{L}}, 1\right) \in \theta_{\left\langle\Gamma^{\mathbf{L}}\right\rangle}$.

In the sequel, when no confusion is possible, we denote by $\phi$ the element $\phi^{\mathbf{L}}$ of $\mathcal{F}_{\mathbf{L}}$ et by $\Gamma$ the subset $\Gamma^{\mathbf{L}}$ of $\mathcal{F}_{\mathbf{L}}$.

Definition 4.4. Assume that $A$ is an $\mathcal{M M V}$-algebra. An algebraic valuation on $A$ is a map $a_{\text {. }}$ : Prop $\rightarrow A$. An algebraic valuation $a$. on $A$ is extended inductively to formulas in the obvious way.

An algebraic model $\langle A, a$.$\rangle is given by an \mathcal{M M V}$-algebra $A$ and an algebraic valuation $a$. on $A$. A formula $\phi$ is true in an algebraic model $\left\langle A, a_{\text {. }}\right\rangle$, in notation $\left\langle A, a_{\text {. }}\right\rangle \models \phi$, if $a_{\phi}=1$.

The following result is a consequence of Lemma 4.3 .

Lemma 4.5. If $\mathbf{L}$ is a modal many-valued logic then a formula $\phi$ belongs to $\mathbf{L}$ if and only if $\phi$ is true in any algebraic model $\langle A, a$.$\rangle where A$ is an $\mathbf{L}$-algebra.

\section{Construction of the Canonical model}

Here comes the first main contribution of the paper. Recall that the variety of MV-algebras is the variety generated by the algebra $\langle[0,1], \oplus, \odot, \neg, 0,1\rangle$ and that an MV-algebra $A$ is simple if and only if it is an isomorphic copy of a subalgebra of $[0,1]$. Moreover, two isomorphic subalgebras of $[0,1]$ are necessarily equal and the isomorphism is the identity. We can thus state the following well known lemma, which will enable us to define a valuation on the canonical model. A filter of an MV-algebra $A$ is maximal if it is maximal among the proper filters of $A$.

Lemma 5.1. A filter $F$ of an $M V$-algebra $A$ is maximal if and only if there is a necessarily unique embedding $f: A / F \rightarrow[0,1]$.

Let us initiate the construction of the canonical model of a modal many-valued logic in the usual way. The universe of the model is the set of the maximal filters of $\mathcal{F}_{\mathbf{L}}$. In order to simplify the expression of our results, it is better to use Lemma 5.1 to identify the set of the maximal filters of $\mathcal{F}_{\mathbf{L}}$ with the set $\mathcal{M V}\left(\mathcal{F}_{\mathbf{L}},[0,1]\right)$ of the homomorphisms of MV-algebras from $\mathcal{F}_{\mathbf{L}}$ to $[0,1]$. The canonical model of a logic $\mathbf{L}$ appears as the canonical KRIPKE model associated to a special algebraic model.

Definition 5.2. If $A$ is an MMV-algebra, the canonical frame $A_{+}$of $A$ is the frame

$$
A_{+}=\left\langle W_{A_{+}}, R^{A_{+}}\right\rangle
$$

where

(1) the universe $W_{A_{+}}$of $A_{+}$is the set $\mathcal{M V}(A,[0,1])$ of the homomorphisms of MV-algebras from $A$ to $[0,1]$; 
(2) the accessibility relation $R^{A_{+}}$is defined by

$$
(u, v) \in R^{A_{+}} \text {if } \forall a \in A(u(\square a)=1 \Rightarrow v(a)=1) .
$$

If $a$. : Prop $\rightarrow A$ is an algebraic valuation, the canonical KRIPKE-model associated to the algebraic model $\left\langle A, a_{.}\right\rangle$is the model $\mathcal{M}_{\langle A, a .\rangle}$ based on the canonical frame of $A$ and defined by

$$
\operatorname{Val}_{\mathcal{M}_{\langle A, a .\rangle}}(u, p)=u\left(a_{p}\right)
$$

for any propositional variable $p$ and any element $u$ of $\mathcal{M V}(A,[0,1])$.

These constructions allow us to associate a canonical frame and a canonical model to a modal many-valued logic $\mathbf{L}$.

Definition 5.3. The canonical frame associated to a modal many-valued logic $\mathbf{L}$ is the canonical frame associated to the $\mathbf{L}$-algebra $\mathcal{F}_{\mathbf{L}}$. The canonical model associated to $\mathbf{L}$ is the canonical model associated to the algebraic model $\left\langle\mathcal{F}_{\mathbf{L}}, a_{\mathbf{.}}\right\rangle$ defined by $a_{p}=p^{\mathbf{L}}$ for any propositional variable $p$.

Note that the definition of the canonical model for $\mathbf{K}_{1}$ coincides with the classical definition of the canonical model for the smallest Boolean modal logic (if we identify any maximal filter $F$ of $\mathcal{F}_{\mathbf{K}_{\mathbf{1}}}$ with the quotient map $\pi_{F}: \mathcal{F}_{\mathbf{K}_{\mathbf{1}}} \rightarrow\{0,1\}$ and if we identify the Boolean valuation map Val : Prop $\rightarrow \mathcal{P}\left(W_{\mathbf{K}_{1}}\right)$ with its characteristic function $)$.

The main result of this section is that the map $\mathrm{Val}_{\mathbf{L}}$ extends to formulas. Before considering the proof of this result, we need the following definition.

Definition 5.4. We denote by $\mathbb{D}$ the subset of $\mathbb{Q}$ that contains the numbers that can be written as a finite sum of powers of 2 . If $r$ is in $\mathbb{D} \cap[0,1]$, we denote by $\tau_{r}$ a composition of the terms $x \oplus x$ and $x \odot x$ such that $\tau_{r}(x)<1$ for every $x \in\left[0, r\left[\right.\right.$ and $\tau_{r}(x)=1$ for every $x \in[r, 1]$. A proof of the existence of such terms can be found in [30 for example. Furthermore, we can always choose $\tau_{r}$ such that $\tau_{r}(x)$ belongs to $\{0,1\}$ for every $x \in \mathrm{E}_{n}$ (but this choice is not independent of $n$ ).

Lemma 5.5. If $\mathbf{L}$ is a modal many-valued logic and if $u, v \in W_{\mathbf{L}}$, then $(u, v) \in R_{\mathbf{L}}$ if and only if $u \circ \square \leq v$.

Proof. The right to left part of the assertion is clear. Let us prove the left to right part and suppose that there is a $\phi$ in $\mathcal{F}_{\mathbf{L}}$, a $v$ in $R_{\mathbf{L}} u$ and an $r$ in $\mathbb{D} \cap[0,1]$ such that $v(\phi)<r \leq u(\square \phi)$. It follows that

$$
\tau_{r}(v(\phi))=v\left(\tau_{r}(\phi)\right)<1 \quad \text { and } \quad 1=\tau_{r}(u(\square \phi))=u\left(\tau_{r}(\square \phi)\right)=u\left(\square \tau_{r}(\phi)\right),
$$

which is a contradiction since $u R_{\mathbf{L}} v$.

Proposition 5.6. Assume that $A$ is an $M M V$-algebra.

(1) If a belongs to $A$ and if $u$ is in $\mathcal{M V}(A,[0,1])$, then $u(\square a)=\bigwedge\{v(a) \mid v \in R u\}$.

(2) If $a$. is an algebraic valuation on $A$, then $\operatorname{Val}_{\left.\mathcal{M}_{\langle A, a}\right\rangle}(u, \phi)=u\left(a_{\phi}\right)$ for any $\phi$ in Form.

(3) If $\mathbf{L}$ is a modal many-valued logic, then $\operatorname{Val}_{\mathcal{M}_{\mathbf{L}}}(u, \phi)=u(\phi)$ for any $\phi$ in Form.

Proof. The second result is a consequence of the first one and the third result is a consequence of the second one. Let us prove the first statement. Assume that $A$ is an MMV-algebra, that $a$ belongs to $A$ and that $u$ belongs to $\mathcal{M V}(A,[0,1])$. We have to prove that

$$
u(\square a)=\bigwedge_{v \in R_{\mathbf{L}} u} v(a) .
$$


The inequality $\leq$ is obtained thanks to Lemma 5.5 .

Now, suppose that the equality does not hold in (5.1), but just the strict inequality $<$. Then, there is an $r$ in $\mathbb{D} \cap[0,1]$ such that

$$
u(\square a)<r \leq \bigwedge_{v \in R_{\mathbf{L}} u} v(a) .
$$

It means that for any $v \in R_{\mathbf{L}} u$, the maximal filter $v^{-1}(1)$ of $\mathcal{F}_{\mathbf{L}}$ contains $\tau_{r}(a)$ and that the filter $\square^{-1} u^{-1}(1)$ does not contain $\tau_{r}(a)$.

But, since $(u, v) \in R_{\mathbf{L}}$ if and only if $\square^{-1} u^{-1}(1) \subseteq v^{-1}(1)$, if follows that the maximal filters of $\mathcal{F}_{\mathbf{L}}$ that contain $\square^{-1} u^{-1}(1)$ are exactly the $v^{-1}(1)$ with $v \in R_{\mathbf{L}} u$, while each of these maximal filters contains $\tau_{r}(a)$. It means that the class of $\tau_{r}(a)$ in $\mathcal{F}_{\mathbf{L}} / \square^{-1} u^{-1}(1)$ is infinitely great, so that $\tau_{r}(a) \oplus \tau_{r}(a)^{m}$ belongs to $\square^{-1} u^{-1}(1)$ for every positive integer $m$. We deduce that

$$
1=u\left(\square\left(\tau_{r}(a) \oplus \tau_{r}(a)^{m}\right)\right) \leq u\left(\tau_{r}(\square a) \oplus\left(\tau_{r}(\square a)\right)^{m}\right),
$$

for any positive integer $m$, so that $u\left(\tau_{r}(\square a)\right)$ is infinitely great in $u\left(\mathcal{F}_{\mathbf{L}}\right)$. Since $u\left(\mathcal{F}_{\mathbf{L}}\right)$ is a subalgebra of $[0,1]$, we obtain that $u\left(\tau_{r}(\square a)\right)=1$, a contradiction.

\section{Completeness for $\mathbf{K}$ and $\mathbf{K}_{n}$}

Proposition 5.6 is the building stone of completeness results for modal many-valued logics and classes of models.

Definition 6.1. Let $\mathbf{L}$ be a modal many-valued logic, let $\Gamma \cup\{\phi\}$ be a set of formulas and $K$ be a class of models. The formula $\phi$ is a local semantic consequence of $\Gamma$ over $K$, in notation $K \models_{\Gamma} \phi$, if for every element $w$ of any model $\mathcal{M}$ of $K$, the formula $\phi$ is true at $w$ in $\mathcal{M}$ whenever $\Gamma$ is true at $w$ in $\mathcal{M}$.

6.1. Modal finitely-valued logics. The first family of systems that admit the many-valued KRIPKE models as a complete semantic is the family of the $\mathfrak{E}_{n}$-valued ones.

Theorem 6.2. If $\Gamma \cup\{\phi\}$ is a set of formulas and if $\mathbf{L}$ is an $E_{n}$-valued logic, then $\Gamma \vdash_{\mathbf{L}} \phi$ if and only if $\phi$ is a local semantic consequence of $\Gamma$ over the class of the models of $\mathbf{L}$.

Proof. The left to right part of the statement is proved in Proposition 6.6 below.

For the right to left part, note that the MV-algebra reduct of the algebra $\mathcal{F}_{\mathbf{L}}$ is a member of $\mathbb{H} \operatorname{SP}\left(\mathrm{E}_{n}\right)=\mathbb{I S P}\left(\mathrm{E}_{n}\right)$ and so is semi-simple. Now, the fact that $\phi$ is a local semantic consequence of $\Gamma$ in the canonical model of $\mathbf{L}$ means that for any $u$ in $\mathcal{M V}\left(\mathcal{F}_{\mathbf{L}},[0,1]\right)$, if $\Gamma \subseteq u^{-1}(1)$ then $\phi \in u^{-1}(1)$. It means equivalently that $\phi$ belongs to any maximal filter extending $\langle\Gamma\rangle$ and so that $\phi$ belongs to $\langle\Gamma\rangle$ thanks to the semi-simplicity of the MV-reduct of $\mathcal{F}_{\mathbf{L}}$.

Note that by considering $n=1$, the preceding proposition boils down to the (strong) completeness result for Boolean modal logic and KRIPKE semantic.

We have announced in section 4 the following result which is an application of the preceding completeness theorem.

Proposition 6.3. If $n$ is a positive integer then $\vdash_{\mathbf{K}_{n}} \square(p \wedge q) \leftrightarrow(\square p \wedge \square q)$.

Moreover, we can simplify the axiomatization 3.1 of $\mathbf{K}_{n}$. We can indeed get rid off the family of axioms that expresses the conservative law of $\square$ with respect to infinitely great elements. This result was first obtained by the authors in the unpublished paper [21]. An other proof was also obtained in [2]. 
Proposition 6.4. If $\mathbf{M V}_{n}$ denotes the $n+1$-valued ŁUKASIEWICZ logic and if $\mathbf{K}_{n}^{\prime}=\mathbf{M V}_{n}+$ $\square(p \rightarrow q) \rightarrow(\square p \rightarrow \square q)+\square(p \oplus p) \leftrightarrow(\square p \oplus \square p)+\square(p \odot p) \leftrightarrow(\square p \odot \square p)$, then $\mathbf{K}_{n}=\mathbf{K}_{n}^{\prime}$.

Proof. In the proof of Proposition 5.6 with $\mathbf{L}=\mathbf{K}_{n}$, we can deduce directly that $u\left(\square \tau_{r}(\phi)\right)=1$ from the fact that $\tau_{r}(\phi)$ is infinitely great in $\mathcal{F}_{\mathbf{K}_{n}} / \square^{-1} u^{-1}(1)$, since $\mathcal{F}_{\mathbf{K}_{n}} / \square^{-1} u^{-1}(1)$ has no non trivial infinitely great element. It means that Proposition 5.6 stands with $\mathbf{L}=\mathbf{K}_{n}^{\prime}$ and that $\vdash_{\mathbf{K}_{n}^{\prime}} \phi$ for any formula $\phi$ that is true in any $\mathbf{E}_{n}$-valued KRIPKE model. We can thus conclude the proof since for any positive integer $m$, the formula $\square\left(p \oplus p^{m}\right) \rightarrow\left(\square p \oplus(\square p)^{m}\right)$ is a tautology.

6.2. Infinitary modal many-valued logics. Unfortunately, when we consider a logic $\mathbf{L}$ that has an algebra $\mathcal{F}_{\mathbf{L}}$ whose MV-reduct is not known to be semi-simple, a completeness result cannot be so easily obtained. This situation occurs for example for the logic $\mathbf{K}$.

For extensions of $\mathbf{K}$, we can obtain completness results by introducing a new infinitary deduction rule.

Definition 6.5. If $\Gamma \cup\{\phi\}$ is a subset of Form and if $\mathbf{L}$ is a modal many-valued logic, we write $\Gamma \vdash_{\mathbf{L}}^{\infty} \phi$ if $\Gamma \vdash_{\mathbf{L}} \phi \oplus \phi^{m}$ for any $m$ in $\mathbb{N}_{0}$.

So, a length of a proof $\Gamma \vdash_{\mathbf{L}}^{\infty} \phi$ is necessarily infinite. Note that if $\Gamma \vdash_{\mathbf{L}} \phi$ then $\Gamma \vdash_{\mathbf{L}}^{\infty} \phi$. We can then state the following completeness result.

Proposition 6.6. If $\mathbf{L}$ is a logic, if $\Gamma \cup\{\phi\}$ is a set of formulas, then $\Gamma \vdash_{\mathbf{L}}^{\infty} \phi$ if and only if $\phi$ is a local semantic consequence of $\Gamma$ in the class of the models of $\mathbf{L}$.

Proof. Assume that $\Gamma \vdash_{\mathbf{L}}^{\infty} \phi$. If $m$ belongs to $\mathbb{N}_{0}$, then there are some $\gamma_{1}, \ldots, \gamma_{r}$ in $\Gamma$ and some $m_{1}, \ldots, m_{r}$ in $\mathbb{N}$ such that $\vdash_{\mathbf{L}}\left(\gamma_{1}^{r_{1}} \odot \cdots \odot \gamma_{r}^{m_{r}}\right) \rightarrow \phi \oplus \phi^{m}$. Since the class of the models of $\mathbf{L}$ forms a sound semantic for $\mathbf{L}$, we can deduce that if $\mathcal{M}=\langle W, R$, Val $\rangle$ is a model of $\mathbf{L}$ and if $\mathcal{M}, u \models \Gamma$ then $\mathcal{M}, u \models \phi \oplus \phi^{m}$ for any $m \in \mathbb{N}_{0}$. We have obtained that the element $\operatorname{Val}(u, \phi)$ is infinitely great in $[0,1]$, which means that it is equal to 1 .

Now, assume that $\phi$ is a local semantic consequence of $\Gamma$ in the class of the many-valued KRIPKE models of $\mathbf{L}$. Since the canonical model of $\mathbf{L}$ is a model of $\mathbf{L}$, it follows that $\phi$ is in any maximal filter that extends $\langle\Gamma\rangle$ in $\mathcal{F}_{\mathbf{L}}$ or equivalently that $\phi$ is infinitely great in $\mathcal{F}_{\mathbf{L}} /\langle\Gamma\rangle$. Thus, the element $\phi \oplus \phi^{m}$ belongs to $\langle\Gamma\rangle$ for any $m$ in $\mathbb{N}_{0}$. We conclude thanks to Lemma 4.3 that $\Gamma \vdash_{\mathbf{L}} \phi \oplus \phi^{m}$ for any $m$ in $\mathbb{N}_{0}$ and eventually that $\Gamma \vdash_{\mathbf{L}}^{\infty} \phi$.

\section{KRIPKE COMPLETENESS AND STRONG KRIPKE COMPLETENESS}

The previous section is concerned with completeness results for modal many-valued logics and classes of KRIPKE models. In this section, we introduce for $\mathrm{E}_{n}$-valued logics two notions of completeness with respect to classes of structures.

7.1. Two types of structures - two types of completeness. The first notion, called strong KRIPKE completeness is a direct adaptation to our many-valued realm of the definition of KRIPKE completeness for Boolean modal logics.

Definition 7.1. A modal $\mathfrak{七}_{n}$-valued logic $\mathbf{L}$ is strongly KRIPKE complete if there is a class $K$ of frames such that $\mathbf{L}=\left\{\phi \in\right.$ Form $\left.\mid \forall \mathfrak{F} \in K, \mathfrak{F} \models_{n} \phi\right\}$.

The reader used to classical modal logic would probably have called KRIPKE complete a logic that we call strongly KRIPKE complete, i.e., a logic which is complete with respect to a class of frames. But, as it will appear after Definition 7.5 , the vocabulary we introduce is coherent. 
In the definition of the KRIPKE semantic for modal $\mathrm{t}_{n}$-valued logics, the many-valued nature of the language that we use appears only in the valuation of the models, not in their underlying frame. One thus may guess that, besides frames, there should exist a type of structures in which this many-valued nature is embodied and that is appropriate for obtaining completeness results. These structures are called $E_{n}$-valued frames. We denote by $\operatorname{div}(n)$ the set of the positive divisors of the element $n$ of $\mathbb{N}$.

Definition 7.2. An $\mathrm{E}_{n}$-valued frame is a structure $\left\langle W,\left\{r_{m} \mid m \in \operatorname{div}(n)\right\}, R\right\rangle$ where

(1) the structure $\langle W, R\rangle$ is a frame,

(2) $r_{m}$ is a subset of $W$ for any $m \in \operatorname{div}(n)$,

(3) $r_{n}=W$ and $r_{m} \cap r_{k}=r_{\operatorname{gcd}(m, k)}$ for any $k, m$ in $\operatorname{div}(n)$,

(4) $R r_{m} \subseteq r_{m}$ for any $m$ in $\operatorname{div}(n)$.

If $\mathfrak{F}=\left\langle W,\left\{r_{m} \mid m \in \operatorname{div}(n)\right\}, R\right\rangle$ is an $\mathfrak{七}_{n}$-valued frame, we denote by $(\mathfrak{F})_{\#}$ its underlying frame $\langle W, R\rangle$. Moreover, a model $\mathcal{M}=\left\langle W^{\prime}, R^{\prime}\right.$, Val $\rangle$ is based on $\mathfrak{F}$ if $\mathcal{M}$ is based on $(\mathfrak{F})_{\#}$ and if $\operatorname{Val}(u, p)$ belongs to $\mathrm{E}_{m}$ for any $u$ in $r_{m}$ and any $m$ in $\operatorname{div}(n)$.

A formula $\phi$ is valid at $u$ in the $E_{n}$-valued frame $\mathfrak{F}$, in notation $\mathfrak{F}, u \models \phi$ if $\mathcal{M}, u \models \phi$ for any model $\mathcal{M}$ based on $\mathfrak{F}$. The formula $\phi$ is valid in $\mathfrak{F}$, in notation $\mathfrak{F}=\phi$, if it is valid at any $u$ in $\mathfrak{F}$.

If $\mathfrak{F}$ is a frame, the trivial $\mathfrak{E}_{n}$-valued frame based on $\mathfrak{F}$ is the $\mathfrak{E}_{n}$-valued frame $\mathfrak{F}^{\prime}=\left\langle W,\left\{r_{m} \mid\right.\right.$ $m \in \operatorname{div}(n)\}, R\rangle$ defined by

(1) $\left(\mathfrak{F}^{\prime}\right) \#=\mathfrak{F}$,

(2) $r_{n}=W$

(3) $r_{m}=\emptyset$ for any $m$ in $\operatorname{div}(n) \backslash\{n\}$.

Roughly speaking, an $\mathrm{E}_{n}$-valued frame is a frame in which we specify in every world $u$ a set of allowed truth values in $u$. With regard to its underlying frame, the satisfaction relation in an $\mathrm{E}_{n}$-valued frame is widened since we have restricted the set of the possible valuations that can be added to the latter structure in order to obtain a model.

Examples of $E_{n}$-valued frames can be obtained by adding structure on the canonical frame associated to an $\mathrm{MMV}_{n}$-algebra $A$, as in the following definition.

Definition 7.3. If $A$ is an $\mathrm{MMV}_{n}$-algebra, the canonical $E_{n}$-valued frame $A_{\times_{n}}$ associated to $A$ is the structure

$$
A_{\times_{n}}=\left\langle W_{A_{\times_{n}}},\left\{r_{m}^{A_{\times_{n}}} \mid m \in \operatorname{div}(n)\right\}, R^{A_{\times_{n}}}\right\rangle,
$$

where

(1) the structure $\left\langle W_{A_{\times_{n}}}, R^{A_{\times_{n}}}\right\rangle$ is the canonical frame associated to $A$,

(2) for any positive divisor $m$ of $n$, the set $r_{m}^{A_{\times_{n}}}$ contains the homomorphisms that are valued in $\mathrm{E}_{m}$ :

$$
r_{m}^{A_{\times_{n}}}=\left\{u \in \mathcal{M V}(A,[0,1]) \mid u(A) \subseteq \mathrm{七}_{m}\right\} .
$$

We prove that canonical $\mathrm{E}_{n}$-valued frames deserve their names, i.e., that $R\left(r_{m}^{A_{\times_{n}}}\right) \subseteq\left(r_{m}^{A \times_{n}}\right)$.

Lemma 7.4. Assume that $A$ belongs to $\mathcal{M M V}_{n}$. The structure $A_{\times_{n}}$ is an $E_{n}$-valued frame. As a consequence, the canonical model associated to an algebraic model $\langle A, a$.$\rangle is based on the$ canonical $E_{n}$-valued frame associated to A.

Proof. Let us assume ad absurdum that there is a $u$ in $r_{m}^{A_{\times_{n}}}$ for which the set $R u \cap A_{\times_{n}} \backslash r_{m}^{A_{\times_{n}}}$ is not empty. Now, since the subalgebras of $\mathfrak{E}_{n}$ are the algebras $\mathbf{E}_{m}$ with $m$ in $\operatorname{div}(n)$, we can 
find an $m^{\prime}$ in $\operatorname{div}(n)$ such that

$$
\frac{1}{m^{\prime}}=\bigwedge\left\{v(x) \mid v \in R u \backslash r_{m}^{A_{\times_{n}}}, x \in A \text { and } v(x) \neq 0\right\} .
$$

Obviously, the integer $m^{\prime}$ is not a divisor of $m$ and we can find a $v \in R u \backslash r_{m}^{A \times_{n}}$ and a $a$ in $A$ such that $v(a)=\frac{1}{m^{\prime}}$.

Let us recall that the universe of $A_{\times_{n}}$ can be equipped with a Boolean topology in such a way that the evaluation map

$$
e_{A}: A \hookrightarrow \prod_{u \in A_{\times_{n}}} u(A): a \mapsto(u(a))_{u \in A_{\times_{n}}}
$$

is a Boolean representation of $A$ such that the set $r_{m}^{A_{\times_{n}}}$ is a closed set for this topology (see Proposition 3.2 in [29] for example). We can thus construct a clopen set $\Omega$ containing $v$ and included in $A_{\times_{n}} \backslash r_{m}^{A_{\times_{n}}}$. Then the element

$$
b=\left.\left.a\right|_{\Omega} \cup 1\right|_{A_{\times_{n}} \backslash \Omega}
$$

belongs to $A$. It follows that

$$
u(\square b)=\bigwedge_{w \in R u} w(b)=\bigwedge_{w \in R u \cap \Omega} w(a)=v(a)=\frac{1}{m^{\prime}}
$$

which is a contradiction since $u \in r_{m}^{A_{\times_{n}}}$.

We can now turn to the definition of KRIPKE completeness.

Definition 7.5. A modal $\mathrm{七}_{n}$-valued logic $\mathbf{L}$ is KRIPKE complete if there is a class $K$ of $\mathrm{E}_{n}$-valued frames such that $\mathbf{L}=\{\phi \in$ Form $\mid \forall \mathfrak{F} \in K, \mathfrak{F} \models \phi\}$.

Our choice in the vocabulary is now totally justified by the fact that the notion of strongly KRIPKE complete logic is definitely stronger than the notion of KRIPKE complete logic. Indeed, if $\mathbf{L}$ is a modal $\mathbf{L}_{n}$-valued logic and if $K$ is a class of frames such that

$$
\mathbf{L}=\left\{\phi \in \text { Form } \mid \forall \mathfrak{F} \in K, \mathfrak{F} \models_{n} \phi\right\},
$$

then it follows obviously that if $K^{\prime}$ denotes the class of the trivial $\mathrm{L}_{n}$-valued frames based on the frames of $K$,

$$
\mathbf{L}=\left\{\phi \in \text { Form } \mid \forall \mathfrak{F} \in K^{\prime}, \mathfrak{F} \models \phi\right\} .
$$

Moreover, the following example proves that there exists a logic that is KRIPKE complete without being strongly KRIPKE complete.

Example 7.6. The logic $\mathbf{L}=\mathbf{K}_{n}+\square(p \vee \neg p)$ is KRIPKE complete but is not strongly KRIPKE complete for $n>1$.

The completeness part is proved in Example 8.28 below.

Let us prove that $\mathbf{L}$ is not strongly KRIPKE complete. Proceed ad absurdum and assume that $K$ is a class of frames such that $\mathbf{L}=\left\{\phi \mid K \models_{n} \phi\right\}$. Then, $K$ contains a frame whose accessibility relation is not empty. Otherwise, the formula $\square \phi$ belongs to $\mathbf{L}$ for any $\phi$, while $\square(p \wedge \neg p)$ does not belong to $\mathbf{L}$.

So, let us denote by $\mathfrak{F}$ a frame with non empty accessibility relation, by $\mathcal{M}=\langle W, R$, Val $\rangle$ a model based on $\mathfrak{F}$ and by $u, v$ two elements of $W$ such that $(u, v) \in R$. Since $\mathcal{M}, u \models \square(p \vee \neg p)$, 
it follows that $\operatorname{Val}(p, v) \in\{0,1\}$. Then, if we denote by $\mathcal{M}^{\prime}=\left\langle W, R, \operatorname{Val}^{\prime}\right\rangle$ the model based on $\mathfrak{F}$ defined by

$$
\operatorname{Val}^{\prime}(q, w)=\left\{\begin{array}{l}
\operatorname{Val}(q, w) \text { if } q \neq p \text { or } w \neq v \\
\frac{1}{n} \text { if } q=p \text { and } w=v
\end{array}\right.
$$

it appears that $\square(p \vee \neg p)$ is not true at $u$ in $\mathcal{M}^{\prime}$, which is the desired contradiction.

As expected, there are algebraic counterpart to these notions of completeness. In order to obtain them, we have to introduce the complex algebras.

7.1.1. Complex algebras. The complex algebras that we introduce are designed to embody the $\mathrm{E}_{n}$-valued modal theory of the various kind of KRIPKE structures. There is no mystery in these constructions: the complex algebra associated to a structure is the algebra of all the possible valuations on that structure.

Definition 7.7. Assume that $\mathfrak{F}=\langle W, R\rangle$ is a frame. The $E_{n}$-complex algebra of $\mathfrak{F}$ is the algebra

$$
\mathfrak{F}^{+n}=\left\langle\mathrm{E}_{n}^{W}, \oplus, \neg, \square_{R}, 0,1\right\rangle,
$$

where the operations $\oplus, \neg, 0,1$ are defined pointwise and the operation $\square_{R}$ is defined by

$$
\square_{R} \alpha(u)=\bigwedge\{\alpha(v) \mid v \in R u\} .
$$

Assume now that $\mathfrak{F}=\left\langle W,\left\{r_{m} \mid m \in \operatorname{div}(n)\right\}, R\right\rangle$ is an $\mathbb{E}_{n}$-valued frame. The $\boldsymbol{E}_{n}$-tight complex algebra of $\mathfrak{F}$ is the algebra

$$
\mathfrak{F}^{\times_{n}}=\left\langle\prod\left\{\mathrm{t}_{s_{u}} \mid u \in W\right\}, \oplus, \neg, \square_{R}, 0,1\right\rangle
$$

where $s_{u}=\operatorname{gcd}\left\{m \in \operatorname{div}(n) \mid u \in r_{m}\right\}$ for any $u$ in $W$ and where the operations are defined exactly as in the definition of the $\mathfrak{E}_{n}$-complex algebra of a frame.

If $K$ is a class of frames we denote by $\mathbf{C m}(K)$ the class of the $\mathrm{E}_{n}$-complex algebras of the elements of $K$ and by $\operatorname{Var}(K)$ the variety generated by $\operatorname{Cm}(K)$. Similarly, if $K$ is a class of $\mathbf{E}_{n}$-valued frames, we denote by $\mathbf{C m}_{n}(K)$ the class of the $\mathbf{E}_{n}$-tight complex algebras of the structures of $K$ and by $\operatorname{Var}_{n}(K)$ the variety generated by $\mathbf{C m}_{n}(K)$. Finally, if $\mathcal{A}$ is a class of $\mathrm{MMV}_{n}$-algebras, we denote by $\operatorname{Str}(\mathcal{A})$ the class of the frames whose $\mathrm{E}_{n}$-complex algebra belongs to $\mathcal{A}$ and we denote by $\operatorname{Str}_{n}(\mathcal{A})$ the class of the $\mathrm{E}_{n}$-valued frames whose $\mathrm{E}_{n}$-tight complex algebra belongs to $\mathcal{A}$.

The following lemma, whose proof is routine, explains how complex algebras embody modal theories.

Lemma 7.8. Assume that $\phi$ is an $\mathcal{L}_{M M V}$-formula.

(1) If $\mathfrak{F}$ is a frame, then $\mathfrak{F}^{+n}$ belongs to $\mathcal{M M V}_{n}$ and $\mathfrak{F} \models_{n} \phi$ if and only if $\mathfrak{F}^{+n} \models \phi=1$.

(2) If $\mathfrak{F}$ is an $E_{n}$-valued frame, then $\mathfrak{F}^{\times_{n}}$ belongs to $\mathcal{M M \mathcal { V } _ { n }}$ and $\mathfrak{F} \models \phi$ if and only if $\mathfrak{F}^{\times_{n}} \models \phi=1$.

We may also note the following result which, albeit obvious, is central for the development of strong KRIPKE completeness results. If $A$ is an MV-algebra, we denote by $\mathfrak{B}(A)$ the Boolean algebra of the idempotent elements of $A$, i.e., the subalgebra of the elements of $A$ that satisfy the equation $x \oplus x=x$.

Lemma 7.9. If $\mathfrak{F}$ is an $\underline{L}_{n}$-valued frame, then $\mathfrak{F}^{\times_{n}}$ is a complete subalgebra of $\mathfrak{F}^{+_{n}}$ and $\mathfrak{B}\left(\mathfrak{F}^{\times_{n}}\right)$ coincides with $\mathfrak{B}\left(\mathfrak{F}^{+_{n}}\right)$.

We now turn to the algebraic counterpart of (strong) KRIPKE completeness. 
Definition 7.10. A variety $\mathcal{A}$ of $\mathrm{MMV}_{n}$-algebras is complete if there is a class $K$ of $\mathrm{七}_{n}$-valued frames such that $\mathcal{A}=\operatorname{Var}_{n}(K)$.

The variety $\mathcal{A}$ is strongly complete if there is a class $K$ of frames such that $\mathcal{A}=\operatorname{Var}(K)$.

Of course, a variety $\mathcal{A}$ is complete if and only if $\mathcal{A}=\operatorname{Var}\left(\operatorname{Str}_{n} \mathcal{A}\right)$, i.e., if and only if $\mathcal{A}$ is generated by its $\mathrm{E}_{n}$-tight complex algebras. It is strongly complete if and only if $\mathcal{A}=\operatorname{Var}(\operatorname{Str} \mathcal{A})$, i.e., if and only if $\mathcal{A}$ is generated by its $\mathrm{七}_{n}$-valued complex algebras.

Once again, a strongly complete variety is a complete variety (since the $\mathrm{E}_{n}$-tight complex algebra of an $\mathrm{E}_{n}$-valued frame is a subalgebra of the $\mathrm{E}_{n}$-valued complex algebra of its underlying frame).

Proposition 7.11. Assume that $\mathbf{L}$ is a modal $E_{n}$-valued logic.

(1) The logic $\mathbf{L}$ is KRIPKE complete if and only if the variety of $\mathbf{L}$-algebras is complete.

(2) The logic $\mathbf{L}$ is strongly KRIPKE complete if and only if the variety of $\mathbf{L}$-algebras is strongly complete.

Proof. (1) Assume that $\mathbf{L}=\bigcap\{\{\phi \in$ Form $\mid \mathfrak{F}=\phi\} \mid \mathfrak{F} \in K\}$ for some class $K$ of $\mathfrak{七}_{n}$-valued frames. Then, the variety $\mathcal{M} \mathcal{M} \mathcal{V}_{\mathbf{L}}$ of $\mathbf{L}$-algebras is the variety of the algebras that satisfy the equations that are valid in $\mathfrak{F}^{\times_{n}}$ for every $\mathfrak{F}$ in $K$. Equivalently, the variety $\mathcal{M M} \mathcal{M} \mathcal{V}_{\mathbf{L}}$ is generated by $K$.

The proof of $(2)$ is similar.

7.2. Completeness through canonicity. KRIPKE completeness results can be obtained through canonicty.

Definition 7.12. A modal $\mathfrak{七}_{n}$-valued logic $\mathbf{L}$ is canonical if $\mathbf{L}$ is valid in the canonical $\mathfrak{七}_{n}$-valued frame associated to $\mathcal{F}_{\mathbf{L}}(X)$ for any set $X$. The logic $\mathbf{L}$ is strongly canonical if $\mathbf{L}$ is valid in the canonical frame associated to $\mathcal{F}_{\mathbf{L}}(X)$ for any set $X$.

Any canonical logic $\mathbf{L}$ is KRIPKE-complete. Indeed, in that case, the logic $\mathbf{L}$ coincides with the set of formulas that are valid in the canonical $\mathfrak{七}_{n}$-valued frame associated to $\mathcal{F}_{\mathbf{L}}(\omega)$. The same line of argument can be used to prove that any strongly canonical logic $\mathbf{L}$ is strongly KRIPKE complete.

Canonicity and strong canonicity can be treated in an algebraic way.

7.2.1. Canonical varieties. Proposition 5.6 allows us to construct two extensions of an $\mathrm{MMV}_{n^{-}}$ algebra $A$.

Lemma 7.13. If $A$ is an $M M V_{n}$-algebra then the algebra $\left(A_{\times_{n}}\right)^{\times_{n}}$ is an extension of $A$.

Proof. We already now that the evaluation map

$$
e_{A}: A \rightarrow\left(A_{\times_{n}}\right)^{\times_{n}}: a \mapsto(u(a))_{u \in A_{\times_{n}}}
$$

is an embedding from the MV-reduct of $A$ to the MV-reduct of $\left(A_{\times_{n}}\right)^{\times_{n}}$. The first item of Proposition 5.6 states that this map is an MMV-homomorphism.

Definition 7.14. Assume that $A$ is an $M_{n}$-algebra. The canonical extension of $A$ is the algebra $\left(A_{\times_{n}}\right)^{\times_{n}}$. The strong canonical extension of $A$ is the algebra $\left(A_{+}\right)^{{ }_{n}}$.

If $\mathcal{A}$ is a variety of $\mathrm{MMV}_{n}$-algebras then $\mathcal{A}$ is canonical if $\mathcal{A}$ contains the canonical extension of any of its algebras. It is strongly canonical if it contains the strong canonical extension of any of its members. 
The reader familiar with the algebraic aspects of classical modal logics should not be surprised by these definitions as we have mimicked the construction of the canonical extension of a Boolean algebra with an operator (as defined in [24] and [25]). Actually, as we will realize later, there is more than a similitude between our approach of canonical extension and the Boolean one. Indeed, these constructions are both two particular cases of the construction of the canonical extension of an expanded bounded distributive lattice. This connection will enable us to give a proof of the following result which states that (strongly) canonical varieties are the algebraic counterpart of (strongly) canonical logics.

Proposition 7.15. Assume that $\mathbf{L}$ is a modal $E_{n}$-valued logic.

(1) The logic $\mathbf{L}$ is canonical if and only if the variety of $\mathbf{L}$-algebras is canonical.

(2) The logic $\mathbf{L}$ is strongly canonical if and only if the variety of $\mathbf{L}$-algebras is strongly canonical.

\section{Canonicity in $\mathcal{M} \mathcal{M} \mathcal{V}_{n}$, a Syntactic approach}

In this section, we approach the problem of canonicity in a syntactic way. Our goal is to produce a class of equations that define canonical varieties.

This famous approach was initiated by JónsSON and TARSKI in their seminal work [24] and [25] about canonical extensions of Boolean algebras with operators. This technique led Jónsson to an algebraic proof of the canonicity of the SAHLQVIST equations (see [23]).

Since then, the theory of canonical extensions was extended to bounded distributive lattices with operators in [14, bounded distributive lattices with monotone maps in [15], bounded distributive expansions in [16] and finally to lattice expansions in [13. These results are the building stones of the syntactic approach of canonicity for various classes of logics including, as we shall see, modal $\mathrm{E}_{n}$-valued logics.

The main result of this section is Theorem 8.26 which is the $\mathbb{E}_{n}$-valued counterpart of the SHALQVIST canonicity result.

8.1. Canonical extensions of bounded distributive lattice expansions. We first recall the theory of canonical extensions for bounded distributive lattice expansions. Our goal is to make this paper self-contained. Our approach is so purely expository. To guide us, we follow the paper [16] in which the proofs of the results we expose can be found.

8.1.1. Canonical extension of bounded distributive lattices. We denote by $\mathcal{D} \mathcal{L}$ the variety of bounded distributive lattices.

Definition 8.1. A complete lattice $A$ is doubly algebraic if it is algebraic and if its order dual $A^{\alpha}$ is algebraic. If $A$ is a complete lattice, we denote respectively by $J^{\infty}(A)$ and $M^{\infty}(A)$ the set of the completely join irreducible elements of $A$ and the set of the completely meet irreducible elements of $A$. The set of the finite joins of elements of $J^{\infty}(A)$ is denoted by $J_{\omega}^{\infty}(A)$ and the set of the finite meets of elements of $M^{\infty}(A)$ is denoted by $M_{\omega}^{\infty}(A)$ (so that 0 belongs to $J_{\omega}^{\infty}(A)$ but does not belong to $J^{\infty}(A)$ and 1 belongs to $M_{\omega}^{\infty}(A)$ but does not belong to $M^{\infty}(A)$ ).

In the variety of bounded distributive lattices, the class of doubly algebraic lattices can be characterized in different ways.

Lemma 8.2. Assume that $A$ is a complete $\mathcal{D} \mathcal{L}$. Then, the following conditions are equivalent.

(1) $A$ is doubly algebraic,

(2) $A$ is algebraic and every element of $A$ is a join of elements of $J^{\infty}(A)$, 
(3) $A$ is completely distributive and every element of $A$ is a join of elements of $J^{\infty}(A)$,

(4) there is a poset $P$ such that $A$ is isomorphic to the lattice of isotone maps from $P$ to the two element chain.

The canonical extension of a $\mathcal{D} \mathcal{L}$ can be described in two different ways. We use the following as a definition.

Definition 8.3. The canonical extension $A^{\sigma}$ of a $\mathcal{D} \mathcal{L} A$ is defined, up to isomorphism, as the lattice of isotone maps from the Priestley dual of $A$ to the two element chain.

Hence, the canonical extension of a bounded distributive lattice is a doubly algebraic lattice. We denote by $\mathcal{D} \mathcal{L}^{+}$the class of doubly algebraic lattices.

It turns out that it is more convenient to characterize the canonical extension $A^{\sigma}$ of a distributive lattice $A$ by properties involving $A$ and $A^{\sigma}$. This characterization requires the following definitions.

Definition 8.4. Assume that $A$ is a sublattice of a $\mathcal{D} \mathcal{L}^{+} B$. The lattice $A$ is a separating sublattice of $B$ if for any $p$ in $J^{\infty}(B)$ and $u$ in $M^{\infty}(B)$ such that $p \leq u$, the interval $[p, u]$ contains an element of $A$.

The sublattice $A$ is compact in $B$ if for any subset $S$ and $T$ of $A$ such that $\bigwedge S \leq \bigvee T$, there are a finite subset $S^{\prime}$ of $S$ and a finite subset $T^{\prime}$ of $T$ such that $\bigwedge S^{\prime} \leq \bigvee T^{\prime}$.

Proposition 8.5. If $A$ is a $\mathcal{D} \mathcal{L}$, then $A$ is a compact separating sublattice of its canonical extension $A^{\sigma}$. Moreover if $B$ is a $\mathcal{D} \mathcal{L}^{+}$that contains $A$ as a separating compact sublattice, then there is a unique isomorphism $f$ from $A^{\sigma}$ to $B$ such that $f\left\llcorner_{A}=\mathrm{id}_{A}\right.$.

We denote by $A^{\alpha}$ the order dual of $A$ for any poset $A$.

Lemma 8.6. If $A_{1}, \ldots, A_{n}$ are $\mathcal{D} \mathcal{L} s$ then

(1) $\left(A_{1}{ }^{\alpha}\right)^{\sigma}$ is equal to $\left(A_{1}^{\sigma}\right)^{\alpha}$,

(2) $\left(A_{1} \times \cdots \times A_{n}\right)^{\sigma}$ is equal to $A_{1}^{\sigma} \times \cdots \times A_{n}^{\sigma}$.

8.1.2. Canonical extensions of $\mathcal{D} \mathcal{L}$-maps. The theory of canonical extension provides two ways to extend maps between $\mathcal{D} \mathcal{L}$ s. These two extensions lead to two definitions of the canonical extension of a bounded distributive lattice expansion (non lattice operations are considered as maps between $\mathcal{D} \mathcal{L} \mathrm{s})$.

To define and study these extensions, we need to introduce two families of topologies.

Definition 8.7. Assume that $A$ is a $\mathcal{D} \mathcal{L}$. A closed element of $A^{\sigma}$ is an element that can be obtained as a meet of elements of $A$. An open element of $A^{\sigma}$ is an element that can be obtained as a join of elements of $A$. We denote by $K\left(A^{\sigma}\right)$ the set of the closed elements of $A^{\sigma}$ and by $O\left(A^{\sigma}\right)$ the set of the open elements of $A^{\sigma}$.

The topologies $\iota^{\uparrow}, \iota^{\downarrow}$ and $\iota$ are defined on $A^{\sigma}$ as the topologies that have respectively for base the sets $[p),(u]$ and $[p) \cap(u]$ where $p$ ranges in $J_{\omega}^{\infty}\left(A^{\sigma}\right)$ and $u$ ranges in $M_{\omega}^{\infty}\left(A^{\sigma}\right)$.

The topologies $\sigma^{\uparrow}, \sigma^{\downarrow}$ and $\sigma$ are defined on $A^{\sigma}$ as the topologies that have respectively for base the sets $[p),(u]$ and $[p) \cap(u]$ where $p$ ranges in $K\left(A^{\sigma}\right)$ and u ranges in $O\left(A^{\sigma}\right)$.

A continuous map $f:\left\langle A^{\sigma}, s\right\rangle \rightarrow\left\langle B^{\sigma}, t\right\rangle$ where $s$ and $t$ are among theses topologies is said to be $(s, t)$-continuous.

Note that $\iota^{\uparrow} \subseteq \sigma^{\uparrow}, \iota^{\downarrow} \subseteq \sigma^{\downarrow}$ and $\iota \subseteq \sigma$. Recall that if $(X, \tau)$ is a topological space, if $Y$ is a dense subset of $X$ and if $B$ is a $\mathcal{D} \mathcal{L}^{+}$, then for any map $f: Y \rightarrow B$, the map $\liminf _{\tau} f$ is defined 
by

and $\lim \sup _{\tau} f$ by

$$
\liminf _{\tau} f: X \rightarrow C: x \mapsto \bigvee\{\bigwedge f(U \cap Y) \mid x \in U \in \tau\}
$$

$$
\lim \sup _{\tau} f: X \rightarrow C: x \mapsto \bigwedge\{\bigvee f(U \cap Y) \mid x \in U \in \tau\}
$$

We can use the density of any $\mathcal{D} \mathcal{L}$ in its canonical extension to define canonical extensions of $\mathcal{D} \mathcal{L}$-maps.

Definition 8.8. If $f: A \rightarrow B$ is a map between two $\mathcal{D} \mathcal{L}$ s $A$ and $B$ then the maps $f^{\sigma}: A^{\sigma} \rightarrow B^{\sigma}$ and $f^{\pi}: A^{\sigma} \rightarrow B^{\sigma}$ are defined by

$$
f^{\sigma}=\liminf _{\sigma} f \quad \text { and } \quad f^{\pi}=\limsup _{\sigma} f .
$$

These maps are called the lower (canonical) extension of $f$ and the upper (canonical) extension of $f$ respectively. The map $f$ is smooth if $f^{\sigma}=f^{\pi}$.

Lemma 8.9. Assume that $f: A \rightarrow B$ is a map between two $\mathcal{D} \mathcal{L} s$ and $B$.

(1) The map $f^{\sigma}$ is the largest $\left(\sigma, \iota^{\uparrow}\right)$-continuous extension of $f$ to $A^{\sigma}$ and $f^{\pi}$ is the smallest $\left(\sigma, \iota^{\downarrow}\right)$-continuous extension of $f$ to $A^{\sigma}$.

(2) The map $f$ is smooth if and only if $f^{\sigma}$ is $(\sigma, \iota)$-continuous.

(3) If $f$ admits a $(\sigma, \iota)$-continuous extension $g: A^{\sigma} \rightarrow B^{\sigma}$ then $f$ is smooth and $f^{\sigma}=g$.

Order preserving properties are translated through canonical extensions to continuity properties.

Definition 8.10. A map $f: A_{1} \times \cdots \times A_{n} \rightarrow B$ between $\mathcal{D} \mathcal{L} \mathrm{s} A_{1}, \ldots, A_{n}, B$ is a (complete) lattice operator or simply a (complete) operator if $f$ is (completely) join preserving in each of its coordinate. It is a dual (complete) operator if $f: A_{1}^{\alpha} \times \cdots \times A_{n}^{\alpha} \rightarrow B^{\alpha}$ is a (complete) operator.

Assume that $\mathcal{L}$ is an expansion of the language $\{\oplus, \odot, \neg, 0,1\}$ with unary operation symbols. We denote by $\mathcal{M V O}{ }_{n}^{\mathcal{L}}$ the variety of $M V_{n}$-algebras with lattice $\mathcal{L}$-operators, i.e., the variety of algebras $A$ over the language $\mathcal{L}$ whose $M V$-reduct belongs to $\mathrm{MV}_{n}$ and such that any unary operation symbol that belongs to $\mathcal{L} \backslash\{\neg\}$ is interpreted as a lattice operator on $A$.

Lemma 8.11. Assume that $f: A \rightarrow B$ is a map between two $\mathcal{D} \mathcal{L} s$ and $B$.

(1) If $f$ is isotone then $f^{\sigma}$ is isotone and is $\left(\sigma^{\uparrow}, \iota^{\uparrow}\right)$-continuous.

(2) If $f$ is an operator then $f^{\sigma}$ is a complete operator and is $\left(\iota^{\uparrow}, \iota^{\uparrow}\right)$-continuous.

(3) If $f$ is join preserving then $f^{\sigma}$ is completely join preserving and is $\left(\sigma^{\downarrow}, \sigma^{\downarrow}\right)$-continuous.

(4) If $f$ is meet preserving and join preserving then $f^{\sigma}$ is $(\sigma, \sigma)$-continuous.

It is also important to compare the canonical extension of a composition of maps with the composition of the canonical extensions of these maps. Indeed, these comparisons are extensively used in the study of stability of equations through canonical extensions.

Lemma 8.12. Assume that $f: B \rightarrow C$ and $g: A \rightarrow B$ are two maps between $\mathcal{D} \mathcal{L} s A, B$ and $C$.

(1) If $f$ and $g$ are isotone maps then $(f g)^{\sigma} \leq f^{\sigma} g^{\sigma}$.

(2) If $f^{\sigma} g^{\sigma}$ is $\left(\sigma, \iota^{\uparrow}\right)$-continuous then $(f g)^{\sigma} \geq f^{\sigma} g^{\sigma}$.

(3) If $f^{\sigma} g^{\sigma}$ is $\left(\sigma, \iota^{\downarrow}\right)$-continuous then $(f g)^{\sigma} \leq f^{\sigma} g^{\sigma}$.

(4) If $f$ is join preserving and meet preserving then $(f g)^{\sigma}=f^{\sigma} g^{\sigma}$.

(5) If $g$ is join preserving and meet preserving then $f^{\sigma} g^{\sigma} \leq(f g)^{\sigma}$.

(6) If $g$ is join preserving, meet preserving and onto then $(f g)^{\sigma}=f^{\sigma} g^{\sigma}$. 
8.1.3. Distributive lattice expansions. The canonical extension of a bounded distributive expansion is built on the canonical extension of its lattice reduct.

Definition 8.13. If $\underline{A}=\left\langle A,\left\{f_{i} \mid i \in I\right\}\right\rangle$ is a bounded distributive expansion of the $\mathcal{D} \mathcal{L} A$, then the canonical extension $\underline{A}^{\sigma}$ of $\underline{A}$ is the algebra $\left\langle A^{\sigma},\left\{f_{i}^{\sigma} \mid i \in I\right\}\right\rangle$ and the dual canonical extension $\underline{A}^{\pi}$ is the algebra $\left\langle A^{\sigma},\left\{f_{i}^{\pi} \mid i \in I\right\}\right\rangle$.

Assume that $A$ is a bounded lattice expansion. The set of the terms $t$ whose term function $t^{A}$ satisfies $\left(t^{A}\right)^{\sigma}=t^{A^{\sigma}}$ is of particular interest. Indeed if $t$ and $s$ are two such terms and if $t^{A}=s^{A}$ it follows that $t^{A^{\sigma}}=\left(t^{A}\right)^{\sigma}=\left(s^{A}\right)^{\sigma}=s^{A^{\sigma}}$. Thus, if the equation $s=t$ is satisfied in $A$, it is also satisfied in $A^{\sigma}$. This piece of argument justifies the following definition.

Definition 8.14. Assume that $\mathcal{L}$ is an expansion of the language $\{\vee, \wedge, 0,1\}$ of bounded distributive lattices. We denote by $\mathcal{D} \mathcal{L} \mathcal{E}_{\mathcal{L}}$ the variety of the bounded distributive lattice $\mathcal{L}$-expansions, i.e., the variety of the algebras over the language $\mathcal{L}$ whose reduct to $\{\vee, \wedge, 0,1\}$ is a $\mathcal{D L}$.

If $A$ belongs to $\mathcal{D} \mathcal{L} \mathcal{E}_{\mathcal{L}}$, an $\mathcal{L}$-term $t$ is expanding on $A$ if $\left(t^{A}\right)^{\sigma} \leq t^{A^{\sigma}}$. It is contracting on $A$ if $\left(t^{A}\right)^{\sigma} \geq t^{A^{\sigma}}$ and stable on $A$ if $\left(t^{A}\right)^{\sigma}=t^{A^{\sigma}}$.

A subvariety $\mathcal{A}$ of $\mathcal{D} \mathcal{L E}_{\mathcal{L}}$ is canonical if it contains the canonical extension of its members and if the canonical extension of an $\mathcal{L}$-homomorphism between two algebras of $\mathcal{A}$ is an $\mathcal{L}$ homomorphism.

Here is an example of expanding term.

Lemma 8.15. Assume that $A$ is a $\mathcal{D} \mathcal{L E}_{\mathcal{L}}$ and that $t$ is an $\mathcal{L}$-term. If for any operation symbol $f$ that occurs in t, the map $f^{A}$ is isotone, then $t$ is expanding on $A$.

Thanks to Theorem 3.15 and Theorem 3.22 in [16, we obtain the following result.

Proposition 8.16. The variety $\mathcal{M V}_{n}$ is canonical. Moreover,

$$
A^{\sigma} \cong \prod\left\{u(A) \mid u \in \mathcal{M V}\left(A, E_{n}\right)\right\}
$$

for any $A$ in $\mathcal{M V}_{n}$.

Corollary 8.17. If $A$ is an $M V_{n}$-algebra, there is a unique isomorphism $\phi: \mathfrak{B}(A)^{\sigma} \rightarrow \mathfrak{B}\left(A^{\sigma}\right)$ with $\phi(a)=a$ for any $a$ in $\mathfrak{B}(A)$. Moreover, this map $\phi$ is an homeomorphism between $\left\langle\mathfrak{B}(A)^{\sigma}, s\left(\mathfrak{B}(A)^{\sigma}\right)\right\rangle$ and $\left\langle\mathfrak{B}\left(A^{\sigma}\right), s\left(A^{\sigma}\right)\left\lfloor_{\mathfrak{B}\left(A^{\sigma}\right)}\right\rangle\right.$ for any $s$ in $\left\{\iota^{\uparrow}, \iota^{\downarrow}, \iota, \sigma^{\uparrow}, \sigma^{\downarrow}, \sigma\right\}$.

Proof. We may for example obtain the isomorphism $\phi$ thanks to Proposition 8.16 and the unicity of $\phi$ follows from Proposition 8.5. Clearly, this isomorphism sends closed, open, completely meet irreducible and completely join irreducible elements to closed, open, completely meet irreducible and completely join irreducible elements respectively and conversely.

Then, if $p$ belongs to $K\left(A^{\sigma}\right)$, it follows that $\mathfrak{B}\left(A^{\sigma}\right) \cap[p)=\mathfrak{B}\left(A^{\sigma}\right) \cap[n . p)$ and $\phi^{-1}\left(\mathfrak{B}\left(A^{\sigma}\right) \cap\right.$ $[p))=\left[\phi^{-1}(n . p)\right)$. Since $n . p=n . \bigwedge\{a \mid p \leq a \in A\}=\bigwedge\{n . a \mid p \leq a \in A\}$ is a closed element of $A^{\sigma}$, we have proved that $\phi:\left\langle\mathfrak{B}(A)^{\sigma}, \sigma^{\uparrow}\left(\mathfrak{B}(A)^{\sigma}\right)\right\rangle \rightarrow\left\langle\mathfrak{B}\left(A^{\sigma}\right), \sigma^{\uparrow}\left(A^{\sigma}\right)\left\lfloor_{\mathfrak{B}\left(A^{\sigma}\right)}\right\rangle\right.$ is continuous.

Now, if $p$ belongs to $K\left(\mathfrak{B}(A)^{\sigma}\right)$, then $\phi([p))=[\phi(p))$ which proves that $\phi^{-1}$ is continuous and so that $\phi$ is an homeomorphism.

We proceed in a similar way for the other topologies.

The next result states that if $A$ is an $\mathrm{MMV}_{n}$-algebra then $A^{\sigma}$ is isomorphic to the $\mathrm{E}_{n}$-tight complex algebra of its $\mathrm{E}_{n}$-valued frame. It means that the two notions of canonical extension introduced for an $\mathrm{MMV}_{n}$-algebra in Definition 7.14 and Definition 8.13 coincide, so that the vocabulary we have introduced is coherent. 
Proposition 8.18. Assume that $A$ is an $M M V_{n}$-algebra. For any $\alpha$ in $A^{\sigma}$ and any $u$ in $\mathcal{M V}\left(A, E_{n}\right)$,

$$
\left(\square^{\sigma} \alpha\right)(u)=\bigwedge\{\alpha(v) \mid v \in R u\} .
$$

Consequently, the algebra $A^{\sigma}$ is isomorphic to $\left(A_{\times_{n}}\right)^{\times_{n}}$ and the variety $\mathcal{M M V}_{n}$ is canonical.

Proof. Let us denote by $\square_{R}$ the operation defined on $A^{\sigma}$ by

$$
\left(\square_{R} \alpha\right)(u)=\bigwedge\{\alpha(v) \mid v \in R u\}
$$

for any $\alpha$ in $A^{\sigma}$ and any $u$ in $\mathcal{M V}\left(A, \succeq_{n}\right)$. We already know that $\square_{R}$ and $\square^{\sigma}$ are extensions of $\square^{A}$. Now, if $p$ is a closed element of $A^{\sigma}$, it follows that

$$
\square^{\sigma} p=\bigwedge\left\{\square a \mid p \leq a \in A^{\sigma}\right\}=\square_{R} p,
$$

since $\square_{R}$ is completely meet preserving. Then, if $\alpha$ belongs to $A^{\sigma}$,

$$
\begin{aligned}
\square^{\sigma} \alpha & =\bigvee\left\{\square^{\sigma} p \mid \alpha \geq p \in K\left(A^{\sigma}\right)\right\} \\
& =\bigvee\left\{\square_{R} p \mid \alpha \geq p \in K\left(A^{\sigma}\right)\right\} .
\end{aligned}
$$

If $u$ belongs to $\mathcal{M V}\left(A, \mathrm{七}_{n}\right)$, we obtain that $\left(\square^{\sigma} \alpha\right)(u)$ is equal to

$$
\bigvee\left\{\left(\square_{R} p\right)(u) \mid \alpha \geq p \in K\left(A^{\sigma}\right)\right\}
$$

so to

and to

$$
\bigvee\left\{\bigwedge\{p(v) \mid(u, v) \in R\} \mid \alpha \geq p \in K\left(A^{\sigma}\right)\right\}
$$

This last element is by definition equal to

$$
\bigwedge\left\{\bigvee\left\{p(v) \mid \alpha \geq p \in K\left(A^{\sigma}\right)\right\} \mid(u, v) \in R\right\}
$$

$$
\bigwedge\{\alpha(v) \mid(u, v) \in R\}
$$

and eventually to $\left(\square_{R} \alpha\right)(u)$.

Let us now assume that $h: A \rightarrow B$ is an MMV-homomorphism. We have to prove that $h^{\sigma}: A^{\sigma} \rightarrow B^{\sigma}$ is an MMV-homomorphism. According to Proposition 8.16, we just have to prove that $h^{\sigma} \square^{\sigma}=\square^{\sigma} h^{\sigma}$. This result is obtained thanks to the sequence of identities

$$
h^{\sigma} \square^{\sigma}=(h \square)^{\sigma}=(\square h)^{\sigma}=\square^{\sigma} h^{\sigma}
$$

in which the second identity is trivial, the first one is obtained by item (4) of Proposition 8.12 and the third one by item (1) and item (5) of the same Proposition.

Now that we know that quotient maps are preserved through canonical extensions, it is a routine argument to prove the first item of Proposition 7.15.

8.2. SAHLQVIST canonicity result for the variety $\mathcal{M M V}_{n}$. SAHLQVIST equations were first introduced in [31] as a family of equations that define canonical logics. The algebraic treatment of this canonicity result was considered in [23. This success lead mathematicians to consider so called "SAHLQVIST equations" in wider contexts (e.g., [17, 19, 9]).

Here, we adapt the classical canonicity result of SAHLQVIST equations to the $\mathfrak{E}_{n}$-valued realm. The algebraic approach makes this adaptation quite painless.

For our purposes, it is important to set the set of primitive operations that we consider to define algebras. So, we are going to denote by $\mathcal{L}_{M M V}$ the set $\{\odot, \vee, \neg, \square, 0,1\}$ where $\odot, \vee$ are binary, the symbols $\neg$ and $\square$ are unary and 0 and 1 are constants. 
The language $\mathcal{L}_{M M V^{d}}$ is the language $\mathcal{L}_{M M V} \cup\{\oplus, \wedge, \diamond\}$, where $\oplus$ and $\wedge$ are binary and $\diamond$ is unary.

The operations $\oplus, \odot, \neg, 0,1$ are intended to be interpreted as the MV-algebra operations. Unless stated otherwise, we do not require any special property on the operation $\square$. But, when we deal with algebras and terms of the language $\mathcal{L}_{M M V^{d}}$, we restrict to algebras that satisfy the following equations

$$
x \wedge y=\neg(\neg x \vee \neg y), \quad x \oplus y=\neg(\neg x \odot \neg y) \quad \text { and } \quad \diamond x=\neg \square \neg x .
$$

More generally, if $g: B_{1} \times \cdots \times B_{k} \rightarrow A$ is a map (a term function for example), then we denote by $g^{d}$ the map

$$
g^{d}: B_{1} \times \cdots \times B_{k} \rightarrow A:\left(x_{1}, \ldots, x_{k}\right) \mapsto \neg g\left(\neg x_{1}, \ldots, \neg x_{k}\right) .
$$

The map $g^{d}$ is called the dual map of $g$, or simply the dual of $g$. The key idea is that by applying equations (8.1) to an $\mathcal{L}_{M M V^{-}}$-term $\tau$, we are able to produce an equivalent $\mathcal{L}_{M M V^{d}}$-term $\tau^{\prime}$ that contains a considerably smaller number of negation symbols. This idea is made clear in the sequel.

The following vocabulary was introduced in [23, 19].

Definition 8.19. Let $\mathcal{L}$ be the language $\mathcal{L}_{M M V}$ or $\mathcal{L}_{M M V^{d}}$. An $\mathcal{L}$-term $\tau$ is

- positive primitive if it is a constant term (i.e., without variable) or if it is equal to $f\left(x_{1}, \ldots, x_{k}\right)$ for a $k$-ary operation $f$ of $\mathcal{L} \backslash\{\neg\}$;

- strictly positive if no variable of $\tau$ is in the scope of a negation symbol (thus, the negation symbols have constant terms as arguments);

- positive if every variable of $\tau$ is in the scope of an even number of negation symbols;

- negative if every variable of $\tau$ is in the scope of an odd number of negation symbols.

If $\mathcal{A}$ is a class of $\mathcal{L}$-algebras, two terms $\tau$ and $\tau^{\prime}$ are said $\mathcal{A}$-equivalent (or simply equivalent if $\mathcal{A}$ is the variety of $\mathcal{L}$-algebras) if the term functions $\tau^{A}$ and $\tau^{\prime A}$ are equal on every algebra $A$ of $\mathcal{A}$ (that satisfies, following our convention, equations 8.1) if $\mathcal{L}=\mathcal{L}_{M M V^{d}}$ ).

The following result is Theorem 6 in [19].

Lemma 8.20. Assume that $\tau$ is an n-ary term over $\mathcal{L}_{M M V}$ or $\mathcal{L}_{M M V^{d}}$.

(1) The term $\tau$ is equivalent to a positive (resp. negative) term if and only if $\tau^{d}$ is equivalent to a positive (resp. negative) term.

(2) If $\sigma_{1}, \ldots, \sigma_{n}$ are terms then $\left(\tau\left(\sigma_{1}, \ldots, \sigma_{n}\right)\right)^{d}=\tau^{d}\left(\sigma_{1}^{d}, \ldots, \sigma_{n}^{d}\right)$.

(3) If $\tau$ is an $\mathcal{L}_{M M V^{d}}$-term then it is equivalent to an $\mathcal{L}_{M M V^{d}}$-term written in standard form, that is an $\mathcal{L}_{M M V^{d}}$-term in which the negation symbols appear next to constant terms or directly next to variables.

Definition 8.21. Let us denote by $\Psi_{0}$ the smallest set of $\mathcal{L}_{M M V}$-terms that contains the positive primitive terms and that is closed under substitution, and by $\Psi$ the smallest set of $\mathcal{L}_{M M V^{d}}$ terms that contains the positive primitive terms and their dual terms and which is closed under substitution.

Once again, the following result can be found in [19.

Proposition 8.22. With the previous notations,

(1) an $\mathcal{L}_{M M V}$-term is equivalent to a strictly positive $\mathcal{L}_{M M V}$-term if and only if it is equivalent to a term of $\Psi_{0}$ 
EXT. ŁUKASIEWICZ LOGICS WITH A MODALITY: ALG. APPROACH TO RELATIONAL SEMANTICS 20

(2) an $\mathcal{L}_{M M V}$-term is equivalent to a positive $\mathcal{L}_{M M V}$-term if and only if it is equivalent to a term of $\Psi$,

(3) an $\mathcal{L}_{M M V}$-term is equivalent to a negative $\mathcal{L}_{M M V}$-term if and only if it is equivalent to the negation of a term of $\Psi$.

We are now ready to define the family of SAHLQVIST equations for the modal $\bigsqcup_{n}$-valued logics.

Definition 8.23. A boxed atom is a variable preceded by a string of boxes.

A SAHLQVIST equation is an equation $\phi \leq \psi$ where

- $\psi$ is a positive term,

- $\phi$ is a term (called a SAHLQVIST antecedent) constructed from boxed atoms, constants and negative terms with lattice operators of $\mathcal{L}_{M M V_{d}}$ (such as $\odot, \oplus, \vee$ and $\wedge$ ).

Note that we allow to construct SAHLQVIST antecedents with MV-operators since these are lattice operators.

Surprisingly, there is a proof of our SAHLQVIST equivalent that is an easy adaptation of the proof of the SAHLQVIST canonicity result for Boolean algebras proposed in [32]. It is the proof that we now develop.

The following result is to $\mathcal{D} \mathcal{L E}_{\mathcal{L}}$ what Theorem 7.20 (ii) in 32 is for expanded Boolean algebras.

Lemma 8.24. Assume that $A$ is a $\mathcal{D} \mathcal{L E}_{\mathcal{L}}$ and that $t$ is an $\mathcal{L}$-term. If every operation symbol that occurs in $t$ is interpreted as a lattice operator on $A$ then $t$ is stable on $A$.

Proof. Lemma 8.15 says that the term $t$ is expanding on $A$. Let us prove by induction on the number of operation symbols that occur in $t$ that $t$ is contracting on $A$. The base case is trivial. Let us then assume that $t=s\left(u_{1}, \ldots, u_{k}\right)$ where $s$ is an operation symbol that is interpreted as a lattice operator on $A$ and where $u_{1}, \ldots, u_{k}$ are terms constructed with connectives that are interpreted as lattice operators on $A$. It follows that

$$
t^{A^{\sigma}}=\left(s^{A}\right)^{\sigma} \circ\left(u_{1}^{A^{\sigma}}, \ldots, u_{k}^{A^{\sigma}}\right) \leq\left(s^{A}\right)^{\sigma} \circ\left(\left(u_{1}^{A}\right)^{\sigma}, \ldots,\left(u_{k}^{A}\right)^{\sigma}\right)
$$

thanks to induction hypothesis. The map $\left(s^{A}\right)^{\sigma}$ is $\left(\iota^{\uparrow}, \iota^{\uparrow}\right)$-continuous since $s^{A}$ is a lattice operator. Similarly, the map $\left(u_{i}^{A}\right)^{\sigma}$ is $\left(\sigma^{\uparrow}, \iota^{\uparrow}\right)$-continuous for any $i$ in $\{1, \ldots, k\}$ since $u_{i}^{A}$ is isotone. Consequently, the map

$$
\left(s^{A}\right)^{\sigma} \circ\left(\left(u_{1}^{A}\right)^{\sigma}, \ldots,\left(u_{k}^{A}\right)^{\sigma}\right)
$$

turns out to be $\left(\sigma^{\uparrow}, \iota^{\uparrow}\right)$ continuous. The result then follows from the second item of Proposition 8.12 .

The following result is to $\mathcal{D} \mathcal{L E}_{\mathcal{L}}$ what Theorem 7.20 (iii) in [32] is for expanded Boolean algebras.

Lemma 8.25. Let $A$ be a $\mathcal{D} \mathcal{L E}_{\mathcal{L}}$ and $t$ be a term. If $t=s\left(u_{1}, \ldots, u_{k}\right)$ where for every operation symbol $f$ that occurs in $s$, the map $f^{A}$ is a lattice operator and where all the connectives in each of the $u_{i}$ are $\wedge$-preserving operation on $A$, then $\tau$ is stable on $A$.

Proof. Lemma 8.15 says that $t$ is expanding on $A$. Let us prove that it is contracting. We have

$$
t^{A^{\sigma}}=s^{A^{\sigma}} \circ\left(u_{1}^{A^{\sigma}}, \ldots, u_{k}^{A^{\sigma}}\right)=\left(s^{A}\right)^{\sigma} \circ\left(\left(u_{1}^{A}\right)^{\sigma}, \ldots,\left(u_{k}^{A}\right)^{\sigma}\right),
$$


thanks to the two preceding lemmas. Then, since each of the $u_{i}$ is $\left(\sigma^{\uparrow}, \iota^{\uparrow}\right)$-continuous and since $\left(s^{A}\right)^{\sigma}=s^{A^{\sigma}}$ is $\left(\iota^{\uparrow}, \iota^{\uparrow}\right)$-continuous, we obtain that $\left(s^{A}\right)^{\sigma} \circ\left(\left(u_{1}^{A}\right)^{\sigma}, \ldots,\left(u_{k}^{A}\right)^{\sigma}\right)$ is $\left(\sigma^{\uparrow}, \iota^{\uparrow}\right)$-continuous and so that

$$
\left(s^{A}\right)^{\sigma} \circ\left(\left(u_{1}^{A}\right)^{\sigma}, \ldots,\left(u_{k}^{A}\right)^{\sigma}\right) \leq\left(s^{A}\left(u_{1}^{A}, \ldots, u_{k}^{A}\right)\right)^{\sigma}
$$

thanks to the second item of Proposition 8.12

The preceding developments lead us to the canonicity of SAHLQVIST equations.

Theorem 8.26. Every SAHLQVIST equation is canonical over the variety $\mathcal{M V O}_{n}^{\mathcal{L}}$.

Proof. We first consider the case of an equation $\phi\left(\beta_{1}, \ldots, \beta_{k}\right) \leq \psi$ where $\psi$ is a positive term, the $\beta_{i}$ are boxed atoms and $\phi$ is constructed only with lattice operators.

Let $A$ be an algebra of $\mathcal{M V O}_{n}^{\mathcal{L}}$. According to the preceding lemma, the term $\phi\left(\beta_{1}, \ldots, \beta_{k}\right)$ is stable on $A$. Now, it follows from Proposition 8.22 and Lemma 8.15 that the term $\psi$ is (equivalent to) an expanding term on $A$. Thus, the term $\phi\left(\beta_{1}, \ldots, \beta_{k}\right) \rightarrow \psi$ is stable on $A$.

Then, consider any SAHLQVIST equation

$$
\phi\left(\beta_{1}, \ldots, \beta_{k}, \psi_{1}^{\prime}, \ldots, \psi_{q}^{\prime}\right) \leq \psi^{\prime}
$$

where the $\beta_{i}$ and $\phi$ are as above, the $\psi_{i}^{\prime}$ are negative and $\psi^{\prime}$ is a positive term. This equation is equivalent to

$$
\neg \psi^{\prime} \odot \phi\left(\beta_{1}, \ldots, \beta_{k}, \psi_{1}^{\prime}, \ldots, \psi_{q}^{\prime}\right)=0 .
$$

Hence, any SAHLQVIST equation is equivalent to an equation of the kind

$$
\phi\left(\beta_{1}, \ldots, \beta_{k}, \neg \psi_{1}, \ldots, \neg \psi_{q}\right)=0
$$

where $\phi$ and the $\beta_{i}$ as are above and the $\psi_{i}$ belongs to $\Psi$. Since $\phi$ is isotone, this equation is in turn equivalent to the quasi-equation

$$
\left(x_{1} \leq \neg \psi_{1}, \ldots, x_{q} \leq \neg \psi_{q}\right) \Rightarrow \phi\left(\beta_{1}, \ldots, \beta_{k}, x_{1}, \ldots, x_{q}\right)=0
$$

where the $x_{i}$ are new variables or, equivalently, to

$$
\left(x_{1} \odot \psi_{1}=0, \ldots, x_{q} \odot \psi_{q}=0\right) \Rightarrow \phi\left(\beta_{1}, \ldots, \beta_{k}, x_{1}, \ldots, x_{q}\right)=0 .
$$

We now introduce a new lattice operator $E$ in the language and interpret it as the global modality:

$$
E^{A}(x)=\left\{\begin{array}{l}
1 \text { if } x>0, \\
0 \text { if } x=0 .
\end{array}\right.
$$

Then, the latter quasi-equation is equivalent to the equation

$$
\phi\left(\beta_{1}, \ldots, \beta_{k}, x_{1}, \ldots, x_{q}\right) \leq E\left(x_{1} \odot \psi_{1}\right) \vee \cdots \vee E\left(x_{q} \odot \psi_{q}\right)
$$

which belongs to the family of equations considered in the first part of the proof.

If we apply Proposition 7.15 to the preceding theorem, we obtain the following completeness result.

Proposition 8.27. If $\phi$ is a formula constructed only with $\vee, \wedge, \oplus, \odot$ and $\diamond$, if the term associated to $\psi$ is positive and if $\beta_{i}$ is a boxed atom or a formula whose associated term is negative for any $i$ in $\{1, \ldots, k\}$ then $\mathbf{K}_{n}+\phi\left(\beta_{1}, \ldots, \beta_{k}\right) \rightarrow \psi$ is a KRIPKE complete logic.

The reader may note that surprisingly, to obtain Proposition 8.27 we had to temporarily allow lattice (non MV-)operators in the language $\mathcal{L}$. 
Example 8.28. The equation $x \oplus x=x$ is canonical since it is equivalent to the SAHLQVIST equation $x \oplus x \leq x$. So, the logic $\mathbf{K}_{n}+(p \oplus p) \leftrightarrow p$ is canonical. This logic is equal to $\mathbf{K}_{1}$ and hence, is not strongly KRIPKE complete. Note that this result can be generalized: if $m$ is a positive divisor of $n$ then the $\mathbf{E}_{n}$-valued logic $\mathbf{K}_{m}=\mathbf{K}_{n}+(m+1) x \leftrightarrow m x+\left\{\left(p x^{p-1}\right)^{m+1} \leftrightarrow\right.$ $(m+1) x^{p} \mid p$ is prime, $p<n$ and $\left.p \notin \operatorname{div}(n)\right\}$ is KRIPKE-complete with respect to the class of the $\mathrm{E}_{n}$-valued frames $\mathfrak{F}=\left\langle W,\left\{r_{k} \mid k \in \operatorname{div}(n)\right\}, R\right\rangle$ that satisfy $r_{k}=W$ if $k$ is a multiple of $m$ and $r_{k}=\emptyset$ otherwise and with an empty accessibility relation $R$.

Similarly, the equation $\square(x \oplus x) \leq \square x$ is a SAHLQvist equation. Hence, the logic $\mathbf{K}_{n}+$ $\square(p \oplus p) \rightarrow \square p$ is canonical. It is easy to realize that this is the logic that we have considered in Example 7.6

\section{Strong Canonicity in $\mathcal{M M V}_{n}$, a Syntactic APPROACH}

The main result of this section is Theorem 9.16 which gives a subfamily of the family of the SAHLQVIST equations made of strongly canonical equations.

Definition 9.1. If $A$ is an $\mathrm{MV}_{n}$-algebra, we denote by $A^{\tau}$ the strong canonical extension of $A$, i.e., the product $\mathrm{MV}_{n}$-algebra $\mathrm{E}_{n}^{A_{+_{n}}}$.

The last item of the following lemma means that the strong canonical extension of an $\mathrm{MV}_{n^{-}}$ algebra $A$ can be defined, up to isomorphism, as the maximal extension of $A$ that is a complete and completely distributive $\mathrm{MV}_{n}$-algebra and whose algebra of idempotents is isomorphic to the canonical extension of the algebra of idempotents of $A$. We will use this fact to extend maps between $\mathrm{MV}_{n}$-algebras to maps between their strong canonical extensions.

Lemma 9.2. If $A$ is an $M V_{n}$-algebra, then

(1) the algebra $A^{\tau}$ is an $M V_{n}$-algebra and $A^{\tau}$ is an extension of $A^{\sigma}$,

(2) the lattice reduct of $A^{\tau}$ is a $\mathcal{D} \mathcal{L}^{+}$,

(3) the Boolean algebras $\mathfrak{B}\left(A^{\tau}\right)$ and $\mathfrak{B}\left(A^{\sigma}\right)$ are isomorphic by a unique isomorphism that fixes $\mathfrak{B}(A)$,

(4) if $B$ is a complete and completely distributive $M V_{n}$-algebra that is an extension of $A$ such that $\mathfrak{B}(B)$ is isomorphic to $\mathfrak{B}\left(A^{\sigma}\right)$ by a necessarily unique isomorphism $l: \mathfrak{B}(B) \rightarrow$ $\mathfrak{B}\left(A^{\sigma}\right)$ fixing $\mathfrak{B}(A)$, then there is a unique embedding $\phi: B \rightarrow A^{\tau}$ that fixes the elements of $\mathfrak{B}\left(A^{\sigma}\right)$ (up to the isomorphism l and the isomorphism of item (3)).

Proof. The proofs of (1), (2), (3) are easy. The map $\phi$ in (4) can be obtained as a composition of the various maps involved.

Let us prove that this map is unique. Assume that $\psi$ satisfies the desired conditions. Then, for any $x$ in $B$, the element $\psi(x)$ is fully determined by the element $\left(\tau_{1 / n}(\psi(x)), \ldots, \tau_{n / n}(\psi(x))\right)$ of $\left(\mathfrak{B}\left(A^{\tau}\right)\right)^{n}$. Now, for any $i$ in $\{1, \ldots, n\}$, we have $\tau_{i / n}(\psi(x))=\psi\left(\tau_{i / n}(x)\right)=l\left(\tau_{i / n}(x)\right)$. Thus, the equality of $\psi$ and $\phi$ follows from the fact that $l$ is unique.

Lemma 9.3. If $A_{1}, \ldots, A_{k}$ are $M V_{n}$-algebras then $\left(A_{1} \times \cdots \times A_{k}\right)^{\tau}=A_{1}^{\tau} \times \cdots \times A_{k}^{\tau}$.

Proof. The proof is direct.

We now introduce a way to extend maps between two $\mathrm{MV}_{n}$-algebras to maps between their strong canonical extensions. Unfortunately, the definition we adopt will not provide an extension for any map. Recall that in an $\mathrm{MV}_{n}$-algebra $A$, any element $x$ is completely determined by the $n$-uple $\left(\tau_{1 / n}(x), \ldots, \tau_{n / n}(x)\right)$ of elements of $\mathfrak{B}(A)$. Hence, if $A$ and $B$ are two MV-algebras and 
if $f^{\prime}: \mathfrak{B}(A) \rightarrow \mathfrak{B}(B)$ is a map, then we can define a map $f: A \rightarrow B$ by defining $f$ as the unique map that satisfies $\tau_{i / n}(f(x))=f^{\prime}\left(\tau_{i / n}(x)\right)$ for any $x$ in $A$ and any $i$ in $\{1, \ldots, n\}$.

This is the way we follow to define an extension $f^{\tau}: A^{\tau} \rightarrow B^{\tau}$ of a map $f: A \rightarrow B$ between two $\mathrm{MV}_{n}$-algebras $A$ and $B$. Thus, the building block of the extension $f^{\tau}$ is a map $f^{\prime}: \mathfrak{B}\left(A^{\tau}\right) \rightarrow \mathfrak{B}\left(B^{\tau}\right)$. Since $\mathfrak{B}\left(A^{\tau}\right)$ is isomorphic to $\mathfrak{B}\left(A^{\sigma}\right)$ and to $\mathfrak{B}(A)^{\sigma}$, we may ride on the existing constructions and define $f^{\prime}$ as one of the maps $\left(f L_{\mathfrak{B}(A)}\right)^{\sigma}$ or $f^{\sigma} L_{\mathfrak{B}\left(A^{\sigma}\right)}$. Of course, in either case, the proposed map $f^{\prime}$ has to be valued in $\mathfrak{B}\left(B^{\tau}\right)$. A natural way to fulfill this condition is to ensure that

$$
\forall x \in A, f(x \oplus x)=f(x) \oplus f(x)
$$

for the first case and that

$$
\forall x \in A^{\sigma}, f^{\sigma}(x \oplus x)=f^{\sigma}(x) \oplus f^{\sigma}(x)
$$

for the second case. Condition $(9.2)$ implies obviously (9.1). Let us denote the unary term $x \oplus x$ by $\tau_{\oplus}$. Now, if $\left(\tau_{\oplus} \circ f\right)^{\sigma}=\tau_{\oplus}^{\sigma} \circ f^{\sigma}$ and $\left(f \circ \tau_{\oplus}\right)^{\sigma}=f^{\sigma} \circ \tau_{\oplus}^{\sigma}$, then, for any map $f$ that satisfies 9.1), we have

$$
f^{\sigma} \circ \tau_{\oplus}^{\sigma}=\left(f \circ \tau_{\oplus}\right)^{\sigma}=\left(\tau_{\oplus} \circ f\right)^{\sigma}=\tau_{\oplus}^{\sigma} \circ f^{\sigma}
$$

and so 9.1 implies 9.2 . So, We are naturally lead to a problem about composition of canonical extensions that can be solved thanks to the tools that we have previously developed.

Lemma 9.4. Assume that $f: A \rightarrow B$ is a map between two $M V_{n}$-algebras $A$ and $B$.

(1) The identity $\tau_{\oplus}^{\sigma} \circ f^{\sigma}=\left(\tau_{\oplus} \circ f\right)^{\sigma}$ and the inequality $f^{\sigma} \circ \tau_{\oplus}^{\sigma} \leq\left(f \circ \tau_{\oplus}\right)^{\sigma}$ are satisfied.

(2) If $f$ is an isotone map then the inequality $f^{\sigma} \circ \tau_{\oplus}^{\sigma} \geq\left(f \circ \tau_{\oplus}\right)^{\sigma}$ is satisfied.

Proof. The identity $\tau_{\oplus}^{\sigma} \circ f^{\sigma}=\left(\tau_{\oplus} \circ f\right)^{\sigma}$ is a consequence of item (4) of Proposition 8.12. The inequality $f^{\sigma} \circ \tau_{\oplus}^{\sigma} \leq\left(f \circ \tau_{\oplus}\right)^{\sigma}$ is an application of item (5) of the same Proposition. The last inequation is a consequence of item (1) of this Proposition.

Recall that the map $f^{\tau}: A^{\tau} \rightarrow B^{\tau}$ that we want to define has to be an extension of $f$. The following lemma states that our methods of construction of $f^{\tau}$ provide an extension of $f$ only if $f$ commutes with $\tau_{\oplus}$ and $\tau_{\odot}$.

Lemma 9.5. Assume that $f: A \rightarrow B$ is a map between two $M V_{n}$-algebras $A$ and $B$.

(1) If $f^{\sigma}\left(\mathfrak{B}\left(A^{\sigma}\right)\right) \subseteq \mathfrak{B}\left(B^{\sigma}\right)$ and if $f^{\prime}: A^{\tau} \rightarrow B^{\tau}$ denotes the map defined by $\tau_{i / n}\left(f^{\prime}(x)\right)=$ $\left.f^{\sigma}\right|_{\mathfrak{B}\left(A^{\sigma}\right)}\left(\tau_{i / n}(x)\right)$ for any $x$ in $A^{\tau}$ and any $i$ in $\{1, \ldots, n\}$ then $\left.f^{\prime}\right|_{A}=f$ if and only if $f\left(\tau_{i / n}(x)\right)=\tau_{i / n}(f(x))$ for any $i$ in $\{1, \ldots, n\}$.

(2) If $f(\mathfrak{B}(A)) \subseteq \mathfrak{B}(B)$ and if $f^{\prime}: A^{\tau} \rightarrow B^{\tau}$ denotes the map defined by $\tau_{i / n}\left(f^{\prime}(x)\right)=$ $\left.f\right|_{\mathfrak{B}(A)} ^{\sigma}\left(\tau_{i / n}(x)\right)$ for any $x$ in $A^{\tau}$ and any $i$ in $\{1, \ldots, n\}$ then $\left.f^{\prime}\right|_{A}=f$ if and only if $f\left(\tau_{i / n}(x)\right)=\tau_{i / n}(f(x))$ for any $i$ in $\{1, \ldots, n\}$.

(3) The map $f$ satisfies $f\left(\tau_{i / n}(x)\right)=\tau_{i / n}(f(x))$ for any $i$ in $\{1, \ldots, n\}$ if and only if $f(x \odot$ $x)=f(x) \odot f(x)$ and $f(x \oplus x)=f(x) \oplus f(x)$ for any $x$ in $A$.

Proof. (1) First assume that $\left.f^{\prime}\right|_{A}=f$. If $x$ belongs to $A$ and $i$ belongs to $\{1, \ldots, n\}$, then $\tau_{i / n}(x)$ belongs to $\mathfrak{B}(A)$ and we obtain that

$$
\tau_{i / n}(f(x))=\tau_{i / n}\left(f^{\prime}(x)\right)=f^{\sigma} L_{\mathfrak{B}\left(A^{\sigma}\right)}\left(\tau_{i / n}(x)\right)=f\left(\tau_{i / n}(x)\right)
$$

since $f^{\sigma} L_{\mathfrak{B}\left(A^{\sigma}\right)}$ is an extension of $f L_{\mathfrak{B}(A)}$. 
Conversely, if $f\left(\tau_{i / n}(x)\right)=\tau_{i / n}(f(x))$ for any $x$ in $A$ and any $i$ in $\{1, \ldots, n\}$ then if $x$ belongs to $A$ and $i$ to $\{1, \ldots, n\}$,

$$
\tau_{i / n}\left(f^{\prime}(x)\right)=f^{\sigma} L_{\mathfrak{B}\left(A^{\sigma}\right)}\left(\tau_{i / n}(x)\right)=f\left(\tau_{i / n}(x)\right)=\tau_{i / n}(f(x)) .
$$

Thus, $f(x)$ and $f^{\prime}(x)$ are equal.

(2) We proceed in a similar way.

(3) The right to left part of the statement is clear. For the left to right part we note that for any $i$ in $\{1, \ldots, n\}$, the terms $\tau_{i / n} \circ \tau_{\odot}$ and $\tau_{i / n} \circ \tau_{\oplus}$ are equivalent on $A$ to a term of $\left\{\tau_{i / n} \mid i \in\{1, \ldots, n\}\right\}$

The preceding lemmas give a justification to the following definition.

Definition 9.6. A map $f: A \rightarrow B$ between two MV-algebras $A$ and $B$ is an idemorphism if $f(x \oplus x)=f(x) \oplus f(x)$ and $f(x \odot x)=f(x) \odot f(x)$ for any $x$ in $A$.

Let us sum up briefly the results we have obtained about the construction of $f^{\tau}$. We want to ride on a map $f^{\prime}: \mathfrak{B}\left(A^{\tau}\right) \rightarrow \mathfrak{B}\left(B^{\tau}\right)$ to define an extension $f^{\tau}: A^{\tau} \rightarrow B^{\tau}$ of a map $f: A \rightarrow B$. We have identified two candidates for the map $f^{\prime}$. These candidates are $f^{\sigma} L_{\mathfrak{B}\left(A^{\sigma}\right)}$ and $f L_{\mathfrak{B}(A)}^{\sigma}$. In both cases, the map $f^{\tau}$ is an extension of $f$ if and only if $f$ is an idemorphism. We now prove that in that case, if in addition $f$ is isotone, then we do not have to choose between $\left.f^{\sigma}\right|_{\mathfrak{B}\left(A^{\sigma}\right)}$ and $f l_{\mathfrak{B}(A)}^{\sigma}$.

Lemma 9.7. If $f: A \rightarrow B$ is an idemorphism between two $M V_{n}$-algebras $A$ and $B$ such that $f^{\sigma}\left(x \oplus^{\sigma} x\right)=f^{\sigma}(x) \oplus^{\sigma} f^{\sigma}(x)$ for any $x$ in $A^{\sigma}$, then $f L_{\mathfrak{B}(A)}^{\sigma}=f^{\sigma}\left\llcorner_{\mathfrak{B}\left(A^{\sigma}\right)}\right.$

Consequently, if $f: A \rightarrow B$ is an isotone idemorphism, then $\left.f\right|_{\mathfrak{B}(A)} ^{\sigma}=f^{\sigma} L_{\mathfrak{B}\left(A^{\sigma}\right)}$.

Proof. We already know that

$$
f^{\sigma}\left\lfloor_{\mathfrak{B}\left(A^{\sigma}\right)}:\left\langle\mathfrak{B}\left(A^{\sigma}\right), \sigma\left(A^{\sigma}\right)\left\lfloor_{\mathfrak{B}\left(A^{\sigma}\right)}\right\rangle \rightarrow\left\langle\mathfrak{B}\left(B^{\sigma}\right), \iota^{\uparrow}\left(B^{\sigma}\right)\left\lfloor_{\mathfrak{B}\left(B^{\sigma}\right)}\right\rangle\right.\right.\right.
$$

is continuous. Up to the isomorphism and homeomorphism $\phi$ of Corollary 8.17 it means that the map

$$
f^{\sigma}\left\lfloor_{\mathfrak{B}\left(A^{\sigma}\right)}:\left\langle\mathfrak{B}(A)^{\sigma}, \sigma\left(\mathfrak{B}(A)^{\sigma}\right)\right\rangle \rightarrow\left\langle\mathfrak{B}(B)^{\sigma}, \iota^{\uparrow}\left(\mathfrak{B}(B)^{\sigma}\right)\right\rangle\right.
$$

is continuous. We conclude that $f^{\sigma} L_{\mathfrak{B}\left(A^{\sigma}\right)} \leq\left. f\right|_{\mathfrak{B}(A)} ^{\sigma}$ since $\left.f\right|_{\mathfrak{B}(A)} ^{\sigma}$ is the largest extension of $\left(f\left\llcorner_{\mathfrak{B}(A)}\right)\right.$ to $\mathfrak{B}(A)^{\sigma}$ that enjoys this property of continuity.

To obtain the other inequality, let us define the map $g: A^{\sigma} \rightarrow B^{\sigma}$ by setting $g(x)=y$ if $f L_{\mathfrak{B}(A)}^{\sigma}\left(\tau_{i / n}(x)\right)=\tau_{i / n}(y)$ for all $i \leq n$. Of course, the maps $g$ and $f L_{\mathfrak{B}(A)}^{\sigma}$ coincide on $\mathfrak{B}\left(A^{\sigma}\right)$. Then, if we prove that $g$ is $\left(\sigma, \iota^{\uparrow}\right)$-continuous, we will obtain that $g \leq f^{\sigma}$ on $\mathfrak{B}\left(A^{\sigma}\right)$ so that $\left.f\right|_{\mathfrak{B}(A)} ^{\sigma} \leq f^{\sigma} L_{\mathfrak{B}\left(A^{\sigma}\right)}$

Let us prove that $g$ is $\left(\sigma, \iota^{\uparrow}\right)$-continuous. Assume that $p$ belongs to $J_{\omega}^{\infty}\left(B^{\sigma}\right)$. We obtain successively that

$$
\begin{aligned}
g^{-1}([p)) & =\bigcap\left\{\left\{x \mid \tau_{i / n}(g(x)) \geq \tau_{i / n}(p)\right\} \mid i \in\{1, \ldots, n\}\right\} \\
& =\bigcap\left\{\left\{x|f|_{\mathfrak{B}(A)}^{\sigma}\left(\tau_{i / n}(x)\right) \geq \tau_{i / n}(p)\right\} \mid i \in\{1, \ldots, n\}\right\} \\
& =\bigcap\left\{\tau_{i / n}^{-1}\left(\left.f\right|_{\mathfrak{B}(A)} ^{\sigma-1}\left(\left[\tau_{i / n}(p)\right)\right)\right) \mid i \in\{1, \ldots, n\}\right\} .
\end{aligned}
$$

Then, since $\tau_{i / n}(p)$ belongs to $J_{\omega}^{\infty}\left(\mathfrak{B}\left(B^{\sigma}\right)\right)=J_{\omega}^{\infty}\left(\mathfrak{B}(B)^{\sigma}\right)$, we can deduce from the $\left(\sigma, \iota^{\uparrow}\right)$ continuity of $f l_{\mathfrak{B}(A)}^{\sigma}$ that $f l_{\mathfrak{B}(A)}^{\sigma-1}\left(\left[\tau_{i / n}(p)\right)\right)$ is an open of $\sigma\left(\mathfrak{B}(A)^{\sigma}\right)$. The conclusion then 
follows from the fact that the map $\tau_{i / n}^{A^{\sigma}}=\left(\tau_{i / n}^{A}\right)^{\sigma}$ is $\left(\sigma\left(A^{\sigma}\right), \sigma\left(\mathfrak{B}(A)^{\sigma}\right)\right)$-continuous since $\tau_{i / n}^{A}$ is both meet and join preserving.

In the applications we develop in the sequel, the maps that we consider are isotone. Thus, there is no need to distinguish $\left.f\right|_{\mathfrak{B}(A)} ^{\sigma}$ from $\left.f^{\sigma}\right|_{\mathfrak{B}\left(A^{\sigma}\right)}$.

Definition 9.8. Assume that $f: A \rightarrow B$ is an idemorphism between two $\mathrm{MV}_{n^{-}}$-algebras $A$ and $B$. The map $f^{\tau}: A^{\tau} \rightarrow B^{\tau}$ is defined by

$$
\forall i \in\{1, \ldots, n\}, \tau_{i / n}^{B^{\tau}}\left(f^{\tau}(x)\right)=f l_{\mathfrak{B}(A)}^{\sigma}\left(\tau_{i / n}^{A^{\tau}}(x)\right),
$$

and is called the strong canonical extension of $f$.

Lemma 9.9. Assume that $f: A \rightarrow B$ is an idemorphism between two $M V_{n}$-algebras $A$ and $B$. Then $f^{\tau}$ is an idemorphism. If $f$ is an isotone map, a lattice operator, a dual lattice operator, a join preserving map or a meet preserving map then $f^{\tau}$ is an isotone map, a lattice operator, a dual lattice operator, a join preserving map, a meet preserving map respectively.

Proof. These results are proved in a similar way. We present the proof for an idemorphism and a lattice operator.

If $f$ is an idemorphism and if $x$ is an element of $A^{\tau}$ then for any $i$ in $\{1, \ldots, n\}$ we obtain successively, if we denote by $l$ the element $\min \left[\frac{i}{2 n}, 1\right] \cap \mathrm{E}_{n}$,

$$
\begin{aligned}
\tau_{i / n}\left(f^{\tau}(x \oplus x)\right) & =f L_{\mathfrak{B}(A)}^{\sigma}\left(\tau_{i / n}(x \oplus x)\right) \\
& =\left.f\right|_{\mathfrak{B}(A)} ^{\sigma}\left(\tau_{l}(x)\right),
\end{aligned}
$$

and

$$
\begin{aligned}
\tau_{i / n}\left(f^{\tau}(x) \oplus f^{\tau}(x)\right) & =\tau_{l}\left(f^{\tau}(x)\right) \\
& =\left.f\right|_{\mathfrak{B}(A)} ^{\sigma}\left(\tau_{l}(x)\right) .
\end{aligned}
$$

We follow that line of argument to prove that $f^{\tau}(x \odot x)=f^{\tau}(x) \odot f^{\tau}(x)$.

Then, let us assume that $f: A_{1} \times \cdots \times A_{k} \rightarrow B$ is an idemorphism and a lattice operator. We prove that $f^{\tau}$ respects the join on the first argument. If $x_{1}$ and $x_{1}^{\prime}$ belong to $A_{1}$ and if $\left(x_{2}, \ldots, x_{k}\right)$ belongs to $A_{2} \times \cdots \times A_{k}$ then for any $i$ in $\{1, \ldots, n\}$,

$$
\begin{aligned}
\tau_{i / n}\left(f^{\tau}\left(x_{1} \vee x_{1}^{\prime}, x_{2}, \ldots, x_{k}\right)\right) & =f l_{\mathfrak{B}(A)}^{\sigma}\left(\tau_{i / n}\left(x_{1} \vee x_{1}^{\prime}, x_{2}, \ldots, x_{k}\right)\right) \\
& =f l_{\mathfrak{B}(A)}^{\sigma}\left(\tau_{i / n}\left(x_{1} \vee x_{1}^{\prime}\right), \ldots, \tau_{i / n}\left(x_{k}\right)\right) \\
& =\left.f\right|_{\mathfrak{B}(A)} ^{\sigma}\left(\left(\tau_{i / n}\left(x_{1}\right) \vee \tau_{i / n}\left(x_{1}^{\prime}\right), \ldots, \tau_{i / n}\left(x_{k}\right)\right)\right)
\end{aligned}
$$

and so $\tau_{i / n}\left(f^{\tau}\left(x_{1} \vee x_{1}^{\prime}, x_{2}, \ldots, x_{k}\right)\right)$ is equal to

$$
\left.f l_{\mathfrak{B}(A)}^{\sigma}\left(\left(\tau_{i / n}\left(x_{1}\right), \ldots, \tau_{i / n}\left(x_{k}\right)\right)\right) \vee f l_{\mathfrak{B}(A)}^{\sigma}\left(\tau_{i / n}\left(x_{1}^{\prime}\right), \ldots, \tau_{i / n}\left(x_{k}\right)\right)\right)
$$

since $f l_{\mathfrak{B}(A)}^{\sigma}$ is a lattice operator. This last element is in turn equal to

$$
\tau_{i / n}\left(f^{\tau}\left(x_{1}, \ldots, x_{k}\right)\right) \vee \tau_{i / n}\left(f^{\tau}\left(x_{1}^{\prime}, \ldots, x_{k}\right)\right)=\tau_{i / n}\left(f^{\tau}\left(x_{1}, \ldots, x_{k}\right) \vee f^{\tau}\left(x_{1}^{\prime}, \ldots, x_{k}\right)\right)
$$

according to the definition of $f^{\tau}$.

Example 9.10. If $A$ is an $\mathrm{MV}_{n^{-}}$-algebra then $\vee^{A}: A \times A \rightarrow A$ and $\wedge^{A}: A \times A \rightarrow A$ are two isotone idemorphisms. It is not hard to check that $\vee^{\tau}=\vee^{A^{\tau}}$ and that $\wedge^{\tau}=\wedge^{A^{\tau}}$. 
Let us also remark that it is possible to regard the negation $\neg$ as an idemorphism. To do so, let us consider $\neg$ as the map $\neg: A^{\alpha} \rightarrow A$. Then, we can prove that the map $\neg^{\tau}: A^{\tau \alpha} \rightarrow A^{\tau}$ is equal to the map $\neg^{A^{\tau}}$. Indeed, the map $\neg^{\tau}: A^{\tau \alpha} \rightarrow A^{\tau}$ is defined for every $x$ in $A^{\tau \alpha}$ by

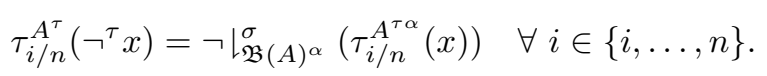

Then, it follows successively that

$$
\begin{aligned}
\left.\neg\right|_{A^{\alpha}} ^{\sigma}\left(\tau_{i / n}^{A^{\tau \alpha}}(x)\right) & =\neg^{A^{\sigma \alpha}}\left(\tau_{i / n}^{A^{\tau \alpha}}(x)\right) \\
& =\neg^{A^{\tau \alpha}}\left(\tau_{i / n}^{A^{\tau \alpha}}(x)\right) \\
& =\tau_{i / n}^{A^{\tau}}\left(\neg^{A^{\tau}}(x)\right) .
\end{aligned}
$$

Proposition 9.11. If $\square$ is a dual $M V$-operator on an $M V_{n}$-algebra $A$ then for any $\alpha$ in $A^{\tau}$ and any $u$ in $A_{+}$

$$
\left(\square^{\tau} \alpha\right)(u)=\bigwedge\{\alpha(v) \mid v \in R u\}
$$

where $R$ denotes the canonical relation associated to $\square$. Consequently, the map $\square^{\tau}$ is a dual $M V$-operator.

Proof. Assume that $\alpha$ belongs to $A^{\tau}$ and $u$ belongs to $A_{+}$. For any $i$ in $\{1, \ldots, n\}$ we obtain successively since $\square$ is isotone that

$$
\begin{aligned}
\left(\tau_{i / n}\left(\square^{\tau} \alpha\right)\right)(u) & =\left(\square^{\sigma}\left(\tau_{i / n}(\alpha)\right)\right)(u) \\
& =\bigwedge\left\{\tau_{i / n}(\alpha)(v) \mid v \in R u\right\} \\
& =\tau_{i / n}(\bigwedge\{\alpha(v) \mid v \in R u\}) .
\end{aligned}
$$

We then obtain that $\square^{\tau}$ is a dual MV-operator thanks to Lemma 7.8 for example.

It is now time to give results about composition of $\tau$-extensions. Once again, our results follow from the results about composition of canonical extensions.

Proposition 9.12. Assume that $f: B \rightarrow C$ and $g: A \rightarrow B$ are two idemorphisms between the $M V_{n}$-algebras $A, B$ and $C$. If $\bowtie$ belongs to $\{\leq, \geq,=\}$ and if $\left.\left.(f g) l_{\mathfrak{B}(A)}^{\sigma} \bowtie f\right|_{\mathfrak{B}(A)} ^{\sigma} g\right|_{\mathfrak{B}(A)} ^{\sigma}$ then $(f g)^{\tau} \bowtie f^{\tau} g^{\tau}$.

Proof. Assume that $\left.\left.\left.(f g)\right|_{\mathfrak{B}(A)} ^{\sigma} \bowtie f\right|_{\mathfrak{B}(A)} ^{\sigma} g\right|_{\mathfrak{B}(A)} ^{\sigma}$. If $x$ belongs to $A^{\tau}$ and $i$ belongs to $\{1, \ldots, n\}$, we obtain successively

$$
\begin{aligned}
\tau_{i / n}\left((f g)^{\tau}(x)\right) & =\left.(f g)\right|_{\mathfrak{B}(A)} ^{\sigma}\left(\tau_{i / n}(x)\right) \\
& \left.\bowtie f\right|_{\mathfrak{B}(A)} ^{\sigma}\left(\left.g\right|_{\mathfrak{B}(A)} ^{\sigma}\left(\tau_{i / n}(x)\right)\right) \\
& =\left.f\right|_{\mathfrak{B}(A)} ^{\sigma}\left(\tau_{i / n}\left(g^{\tau}(x)\right)\right) \\
& =\tau_{i / n}\left(f^{\tau} g^{\tau}(x)\right)
\end{aligned}
$$

which concludes the proof.

In order to determine if a variety $\mathcal{A}$ of $\mathrm{MMV}_{n}$-algebras contains the $\tau$-extension of any of its element, it is useful to prove that if $B$ is a quotient of the $\mathcal{A}$-algebra $A$, then $B^{\tau}$ is a quotient of $A^{\tau}$. We first consider the more general problem of the conservation of homomorphisms: if $f: A \rightarrow B$ is an homomorphism between two $\mathcal{A}$-algebras $A$ and $B$, can we deduce that $f^{\tau}: A^{\tau} \rightarrow B^{\tau}$ is an homomorphism?

We have to keep in mind that, unlike the case of canonical extension, the operation $\oplus^{A^{\tau}}$ is not obtained as the $\tau$-extension of $\oplus^{A}$ since it is not an idemorphism. 
The result we obtain is more general than needed.

Definition 9.13. An algebra $A$ is an $M V_{n}$-algebra with $\mathcal{L}$-idemorphisms (resp. $M V_{n}$-algebra with $\mathcal{L}$-lattice idemorphisms) if it is an $\mathcal{L}$-algebra such that $\langle A, \oplus, \odot, \neg, 0,1\rangle$ is an $\mathrm{MV}_{n}$-algebra and if any operation $g$ of $\mathcal{L} \backslash \mathcal{L}_{M V}$ is interpreted as an idemorphism (resp. and as a lattice operator) $g^{A}$ on the MV-algebra reduct of $A$.

If $A$ is an $\mathrm{MV}_{n}$-algebra with $\mathcal{L}$-idemorphisms, the strong canonical extension $A^{\tau}$ of $A$ is defined as the $\mathcal{L}$-algebra whose MV-reduct is the strong canonical extension of the MV-reduct of $A$ and that satisfies $g^{A^{\tau}}=\left(g^{A}\right)^{\tau}$ for any operation symbol $g$ in $\mathcal{L} \backslash \mathcal{L}_{M V}$.

So, in the construction of strong canonical extensions of $\mathrm{MV}_{n}$-algebras, the algebras are considered more as expanded MV-algebras than expanded $\mathcal{D} \mathcal{L}$ s.

An MMV-algebra is an example of an MV-algebra with a unary lattice idemorphism.

In the sequel, if $f: A \rightarrow B$ is a map, we denote by $f^{[k]}$ the map $f^{[k]}: A^{k} \rightarrow B^{k}:\left(a_{1}, \ldots, a_{k}\right) \mapsto$ $\left(f\left(a_{1}\right), \ldots, f\left(a_{k}\right)\right)$.

Lemma 9.14. Assume that $A$ and $B$ are $M V_{n}$-algebras with $\mathcal{L}$-lattice idemorphisms. For any $\mathcal{L}$-homomorphism $f: A \rightarrow B$ the map $f^{\tau}: A^{\tau} \rightarrow B^{\tau}$ is an $\mathcal{L}$-homomorphism.

Proof. First, assume that $g$ is a $k$-ary operation of $\mathcal{L} \backslash \mathcal{L}_{M V}$ interpreted as a lattice idemorphism on the algebras $A^{k}$ and $B^{k}$. If $\left(x_{1}, \ldots, x_{k}\right)$ belongs to $\left(A^{\tau}\right)^{k}$ and $i$ belongs to $\{1, \ldots, n\}$, we obtain successively on the one hand that

$$
\begin{aligned}
\tau_{i / n}\left(\left(f^{\tau}\left(g^{A}\right)^{\tau}\left(x_{1}, \ldots, x_{k}\right)\right)\right) & =\left(\left.\left.f\right|_{\mathfrak{B}(A)} ^{\sigma} g^{A}\right|_{\mathfrak{B}(A)^{k}} ^{\sigma}\right)\left(\tau_{i / n}\left(x_{1}\right), \ldots, \tau_{i / n}\left(x_{k}\right)\right) \\
& =\left(\left.\left.g^{B}\right|_{\mathfrak{B}(B)^{k}} ^{\sigma} f\right|_{\mathfrak{B}(A)} ^{\sigma[k]}\right)\left(\tau_{i / n}\left(x_{1}\right), \ldots, \tau_{i / n}\left(x_{k}\right)\right) \\
& =\tau_{\frac{i}{n}}\left(\left(g^{B}\right)^{\tau} f^{\tau[k]}\left(x_{1}, \ldots, x_{k}\right)\right) .
\end{aligned}
$$

Let us now prove that $f^{\tau}\left(x \oplus^{A^{\tau}} y\right)=f^{\tau}(x) \oplus^{B^{\tau}} f^{\tau}(y)$ for any $x$ and $y$ in $A^{\tau}$. Let $i$ be an element of $\{1, \ldots, n\}$. The equation

$$
\tau_{i / n}(x \oplus y)=\tau_{i / n}(x) \vee\left(\tau_{(i-1) / n}(x) \wedge \tau_{1 / n}(y)\right) \vee \cdots \vee\left(\tau_{1 / n}(x) \wedge \tau_{(i-1) / n}(y)\right) \vee \tau_{i / n}(y)
$$

(where $\tau_{0}$ is defined as the constant term 1 ) is satisfied in the variety of $\mathrm{MV}_{n}$-algebras. If $x$ and $y$ belong to $A^{\tau}$, then $\tau_{i / n}\left(f^{\tau}(x) \oplus f^{\tau}(y)\right)$ is equal, thanks to equation (9.3), to

$\tau_{i / n}\left(f^{\tau}(x)\right) \vee\left(\tau_{(i-1) / n}\left(f^{\tau}(x)\right) \wedge \tau_{1 / n}\left(f^{\tau}(y)\right)\right) \vee \cdots \vee\left(\tau_{1 / n}\left(\left(f^{\tau}(x)\right) \wedge \tau_{(i-1) / n}\left(\left(f^{\tau}(y)\right)\right) \vee \tau_{i / n}\left(\left(f^{\tau}(y)\right)\right.\right.\right.$,

which is in turn equal, by definition of $f^{\tau}$, to

$f^{\sigma}\left(\tau_{i / n}(x)\right) \vee\left(f^{\sigma}\left(\tau_{(i-1) / n}(x)\right) \wedge f^{\sigma}\left(\tau_{1 / n}(y)\right)\right) \vee \cdots \vee\left(f^{\sigma}\left(\tau_{1 / n}(x)\right) \wedge f^{\sigma}\left(\tau_{(i-1) / n}(y)\right)\right) \vee f^{\sigma}\left(\tau_{i / n}(y)\right)$.

Then, since $f^{\sigma}: A^{\sigma} \rightarrow B^{\sigma}$ is an homomorphism of MV-algebras, this last element is equal to

$$
f^{\sigma}\left(\tau_{i / n}(x) \vee\left(\tau_{(i-1) / n}(x) \wedge \tau_{1 / n}(y)\right) \vee \cdots \vee\left(\tau_{1 / n}(x) \wedge \tau_{(i-1) / n}(y)\right) \vee \tau_{i / n}(y)\right),
$$

i.e., to

$$
f^{\sigma}\left(\tau_{i / n}(x \oplus y)\right)=\tau_{i / n}\left(f^{\tau}(x \oplus y)\right) .
$$

We proceed in a similar way to prove that $f^{\tau}(\neg x)=\neg f(x)$ for any $x$ in $A^{\tau}$.

Note that thanks to the preceding result, it is now easy to give a proof of the second item of Proposition 7.15 .

Lemma 9.15. Assume that $A$ is an $M V_{n}$-algebra with $\mathcal{L}$-operators. 
(1) If $t$ is an $\mathcal{L}$-term constructed with operations that are interpreted as isotone idemorphisms on $A$ then $t^{A^{\tau}} \geq\left(t^{A}\right)^{\tau}$.

(2) If $t$ is an $\mathcal{L}$-term constructed with operations that are interpreted as lattice idemorphisms on $A$ then $t^{A^{\tau}}=\left(t^{A}\right)^{\tau}$.

(3) If $t=s\left(u_{1}, \ldots, u_{k}\right)$ is an $\mathcal{L}$-term where for every operation symbol $f$ that appears in $s$ the map $f^{A}$ is a lattice idemorphism and where all the operations in each of the $u_{i}$ are interpreted as meet preserving idemorphisms, then $\left(t^{A^{\tau}}\right)=\left(t^{A}\right)^{\tau}$.

Proof. The proofs are done by induction on the number of connectives in $t$ with the help of Proposition 9.12 and the corresponding results for canonical extensions.

Theorem 9.16. Assume that $\phi \leq \psi$ is a SAHLQVIST equation over the language $\mathcal{L}_{M M V^{d}}$ where

- the term $\psi$ is constructed only with the operations $\neg, \vee, \wedge$, constants, modalities and dual modalities,

- the term $\phi$ is constructed from boxed atoms, constants with the operations $\vee, \wedge$ and modalities.

The equation $\phi \leq \psi$ is strongly canonical and thus the logic $\mathbf{K}_{n}+\phi \rightarrow \psi$ is a KRIPKE-complete logic.

Example 9.17. The equations $\square p \rightarrow p, \square p \rightarrow \square \square p, p \rightarrow \square \diamond p$ are all strongly canonical and hence define strongly KRIPKE complete logics.

\section{REFERENCES}

[1] Patrick Blackburn, Maarten de Rijke, and Yde Venema, Modal logic, Cambridge Tracts in Theoretical Computer Science, vol. 53, Cambridge University Press, Cambridge, 2001.

[2] Felix Bou, Francesc Esteva, Lluis Godo, and Ricardo Rodriguez, On the minimum Many-Valued modal logic over a finite residuated lattice, 0811.2107 (2008).

[3] Xavier Caicedo and Ricardo O. Rodriguez, Standard Gödel modal logics, Studia Logica 94 (2010), no. 2, 189-214. MR MR2602572

[4] Alexander Chagrov and Michael Zakharyaschev, Modal logic, Oxford Logic Guides, vol. 35, The Clarendon Press Oxford University Press, New York, 1997, Oxford Science Publications.

[5] C. C Chang, Algebraic analysis of many valued logics, Transactions of the American Mathematical Society 88 (1958), 467-490.

[6] - A new proof of the completeness of the Eukasiewicz axioms, Transactions of the American Mathematical Society 93 (1959), 74-80.

[7] Brian F Chellas, Modal logic. An introduction, Cambridge University Press, Cambridge, 1980.

[8] Roberto L. O Cignoli, Itala M. L D'Ottaviano, and Daniele Mundici, Algebraic foundations of many-valued reasoning, Trends in Logic-Studia Logica Library, vol. 7, Kluwer Academic Publishers, Dordrecht, 2000.

[9] Maarten de Rijke and Yde Venema, Sahlqvist's theorem for boolean algebras with operators with an application to cylindric algebras, Studia Logica. An International Journal for Symbolic Logic 54 (1995), no. 1, 61-78.

[10] Melvin Fitting, Many-valued modal logics, Fundamenta Informaticae 15 (1991), no. 3-4, 235-254.

[11] _ Many-valued modal logics. II, Fundamenta Informaticae 17 (1992), no. 1-2, 55-73.

[12] _ Tableaus for many-valued modal logic, Studia Logica. An International Journal for Symbolic Logic 55 (1995), no. 1, 63-87.

[13] Mai Gehrke and John Harding, Bounded lattice expansions, Journal of Algebra 238 (2001), no. 1, 345-371.

[14] Mai Gehrke and Bjarni Jónsson, Bounded distributive lattices with operators, Mathematica Japonica 40 (1994), no. 2, 207-215.

[15] _ Monotone bounded distributive lattice expansions, Mathematica Japonica 52 (2000), no. 2, 197-213.

[16] _ Bounded distributive lattice expansions, Mathematica Scandinavica 94 (2004), no. 1, 13-45.

[17] Mai Gehrke, Hideo Nagahashi, and Yde Venema, A Sahlqvist theorem for distributive modal logic, Annals of Pure and Applied Logic 131 (2005), no. 1-3, 65-102. 
[18] Joan Gispert and Daniele Mundici, MV-algebras: a variety for magnitudes with archimedean units, Algebra Universalis 53 (2005), no. 1, 7-43.

[19] S. Givant and Y. Venema, The preservation of Sahlqvist equations in completions of boolean algebras with operators, Algebra Universalis 41 (1999), no. 1, 47-84.

[20] Siegfried Gottwald, A treatise on many-valued logics, Studies in Logic and Computation, vol. 9, Research Studies Press Ltd., Baldock, 2001.

[21] Georges Hansoul and Bruno Teheux, Completeness results for many-valued Eukasiewicz modal systems and relational semantics, arXiv:math/0612542 (2006).

[22] Petr Hájek, Metamathematics of fuzzy logic, Trends in Logic-Studia Logica Library, vol. 4, Kluwer Academic Publishers, Dordrecht, 1998.

[23] Bjarni Jónsson, On the canonicity of Sahlqvist identities, Studia Logica. An International Journal for Symbolic Logic 53 (1994), no. 4, 473-491.

[24] Bjarni Jónsson and Alfred Tarski, Boolean algebras with operators. I, American Journal of Mathematics 73 (1951), 891-939.

[25] B Boolean algebras with operators. II, American Journal of Mathematics 74 (1952), 127-162.

[26] Saul A Kripke, Semantical analysis of modal logic. I. normal modal propositional calculi, Zeitschrift $\mathrm{f} \backslash$ "ur Mathematische Logik und Grundlagen der Mathematik 9 (1963), 67-96.

[27] Jan Łukasiewicz, O logice trójwarto'sciowej, Ruch Filozoficny 5 (1920), 170-171.

[28] Jan Łukasiewicz and Tarski Alfred, Untersuchungen über den ausagenkalkül, Compte Rendus Séances Société des Sciences et Lettres Varsovie 23 (1930), 30-50.

[29] Philippe Niederkorn, Natural dualities for varieties of $M V$-algebras. I, Journal of Mathematical Analysis and Applications 255 (2001), no. 1, 58-73.

[30] Pascal Ostermann, Many-valued modal propositional calculi, Zeitschrift für Mathematische Logik und Grundlagen der Mathematik 34 (1988), no. 4, 343-354.

[31] Henrik Sahlqvist, Completeness and correspondence in the first and second order semantics for modal logic, Proceedings of the Third Scandinavian Logic Symposium (Univ. Uppsala, Uppsala, 1973) (Amsterdam), North-Holland, 1975, pp. 110-143. Stud. Logic Found. Math., Vol. 82.

[32] Yde Venema, Algebras and coalgebras, Studies in logic and practical reasoning, Elsevier, 2006.

Département de Mathématiques, Université de Liège, 12, Grande Traverse, 4000 Liège, Belgium. E-mail address: g.hansoul@ulg.ac.be

E-mail address: B.Teheux@alumni.ulg.ac.be 